\title{
Isotopic effects in partially deuterated piezoelectric crystals of Rochelle salt
}

\author{
R.R.Levitskii ${ }^{1}$, I.R.Zachek ${ }^{2}$, A.P.Moina ${ }^{1}$, A.Ya.Andrusyk ${ }^{1}$ \\ ${ }^{1}$ Institute for Condensed Matter Physics, National Academy of Sciences of \\ Ukraine, 1 Svientsitskii Str., 79011 Lviv, Ukraine \\ 2 State University “Lvivs'ka Politekhnika”, 12 Bandera Str., 79013, Lviv, \\ Ukraine
}

Received February 4, 2004

\begin{abstract}
We develop a theory for dielectric, piezoelectric, and elastic properties of partially deuterated (quenched disorder) crystals of Rochelle salt with taking into account the piezoelectric coupling. Results of numerical calculations are presented for a completely deuterated Rochelle salt and compared with available experimental data. Isotopic effect is explored within the mean crystal approximation, as well as within the developed theory using different trial sets of the fitting parameters. Theory predictions are given for the temperature and composition dependences of the calculated characteristics for partially deuterated crystals.
\end{abstract}

Key words: Rochelle salt, deuteration, piezoelectric effect

PACS: $77.22 . \mathrm{Gm}, 77.65 . \mathrm{Bn}, 77.80 . \mathrm{Bh}$

\section{Introduction}

Rochelle salt (double sodium-potassium tartrate $\mathrm{NaKC}_{4} \mathrm{H}_{4} \mathrm{O}_{6} \cdot 4 \mathrm{H}_{2} \mathrm{O}$ ) has two Curie points. The ferroelectric phase exists in a rather narrow temperature interval from $T_{\mathrm{C} 1}=255 \mathrm{~K}$ to $T_{\mathrm{C} 2}=297 \mathrm{~K}\left(T_{\mathrm{C} 1}=251 \mathrm{~K}, T_{\mathrm{C} 2}=308 \mathrm{~K}\right.$ in deuterated crystals dRs). Spontaneous polarization is directed along the $a$ axis; it is accompanied by a spontaneous shear strain $\varepsilon_{4}$. Crystal structure is monoclinic $C_{2}^{2}\left(P 2_{1}\right)$ in the ferroelectric phase and orthorhombic $D_{2}^{3}\left(P 2_{1} 2_{1} 2_{1}\right)$ in the paraelectric phases.

According to the classical concepts, based on structural data of Frazer et al. [1], the phase transitions in Rochelle salt are pure order-disorder ones. The ferroelectric polarization used to be attributed to rotation of hydroxyl groups of tartrate complexes $\mathrm{OH}_{5}$ between two equilibrium positions (see [2]). The actual situation is far more complicated, and the mechanism of the phase transitions in Rochelle salt remains rather obscure. More recent neutron scattering data indicate that the $\mathrm{OH}_{5}$ hydroxyl groups do not perform any orientational motion and therefore play little 
role in the phase transition, at least in deuterated Rochelle salt [3,4]. Furthermore, experimental facts suggest that the phase transitions in Rochelle salt are displacive $[5,6]$ ones or of mixed order-disorder and displacive type $[7,8]$. According to $X$-ray scattering experiments [9], spontaneous polarization in Rochelle salt is created by cooperative displacements of tartrate molecules and water molecules in a frame of $\mathrm{K}$ and $\mathrm{Na}$ ions. Recently Hlinka, Petzelt et al. [10], based on their $X$-ray diffraction data, proposed that it is the order-disorder motion of $\mathrm{OH}_{9}$ and $\mathrm{OH}_{10}$ groups, coupled with the displacive vibrations of $\mathrm{OH}_{8}$ groups that is responsible for the phase transitions in Rochelle salt, as well as for the spontaneous polarization. So far it has not been definitively established the motion of which atoms is the order-disorder one.

The most successful microscopic model for Rochelle salt was proposed by Mitsui in [11]. It is based on the assumption that ordering structure elements move in asymmetric double well potentials. The dipoles form two co-penetrating sublattices, with the local potentials which are the mirror reflections of each other. Therefore, even though the dipoles in each sublattice are always ordered (non-zero sublattice polarization), there may be no total polarization at certain temperatures. Later $[12,13]$ the model was reformulated in terms of pseudospin operators; the model itself and its modifications were used for a description of Rs, dRs, $\mathrm{RbHSO}_{4}, \mathrm{NH}_{4} \mathrm{HSO}_{4}$ and other crystals. It should be mentioned here that crystals of $\mathrm{RbHSO}_{4}$ undergo a single second order phase transition into the ferroelectric phase, whereas in $\mathrm{NH}_{4} \mathrm{HSO}_{4}$ the ferroelectric phase exists in a narrow temperature interval but, in contrast to the situation with Rs, the lower phase transition is of the first order.

Thermodynamic characteristics of the Mitsui model within the mean field approximation (MFA) were calculated in [13-15]. Tunneling of ordering structure elements was taken into account in $[14,15]$. Relaxation phenomena in the crystals described by the Mitsui model were explored within the stochastic Glauber model [16] in [17] and Bloch equation method in [18]. Within the MFA the relaxation times for Rs and dRs were calculated. It should be noted that in the mentioned papers only one or very few physical characteristics of Rs and dRs were fitted to experimental data, whereas the other characteristics were not considered. Naturally, such a method cannot prove the adequacy of the Mitsui model to Rochelle salt crystals, since it is quite simple to get a good fit to experiment, when only selected characteristics are calculated, and a sufficient number of the free parameters is invoked.

Ferroelectric crystals $\mathrm{RbHSO}_{4}$ and $\mathrm{NH}_{4} \mathrm{HSO}_{4}$ within the Mitsui model were studied in [19-21]. In these crystals the phase transitions are associated with ordering of sulphate groups (see [19]). Thermodynamic characteristics were calculated within the MFA and two-particle appoximation and compared with experimental data. Overall a fair description of the data was obtained for $\mathrm{RbHSO}_{4}$. As for $\mathrm{NH}_{4} \mathrm{HSO}_{4}$, in [21] it was shown that within the MFA for the Mitsui model it is impossible to describe the low-temperature phase transition in it. At the same time in [20] the temperature curve of spontaneous polarization in $\mathrm{NH}_{4} \mathrm{HSO}_{4}$ was described, assuming that certain interaction parameters are temperature dependent.

Possibility to describe several systems with different numbers of phase transitions 
within a Mitsui model indicates an importance of exploring the phase diagram of the model. One of the first approximate phase diagrams was presented in [14]; tunneling was not taken into account. A more precise phase diagram with zero tunneling was constructed in [22]. Regions with three phase transitions were shown to exist. In [22] a Mitsui model with tunneling was considered as well. Within the MFA it was shown that with non-zero tunneling there are such values of the model parameters when the system undergoes a low temperature first order transition and a high temperature second order transition, with the ferroelectric phase in between, as observed in $\mathrm{NH}_{4} \mathrm{HSO}_{4}$. The most thorough analysis of the phase diagram of the Mitsui model was obtained in [23] within the MFA both with and without tunneling.

A more consistent attempt to describe the Rs, dRs and $\mathrm{RbHSO}_{4}$ crystals within the Mitsui model was undertaken in [24-26]. The free energy, spontaneous polarization, entropy, specific heat, static and dynamic dielectric permittivity (the latter within the stochastic Glauber model) of the Mitsui model were calculated. For the first time there have been found such values of the theory parameters which provided a more or less satisfactory description of several characteristics of Rs, dRs, and $\mathrm{RbHSO}_{4}$. The remaining contradictions between theory and experiment and doubts in reliability of some experimental data for $\mathrm{Rs}$, dRs, and $\mathrm{RbHSO}_{4}$ were approached in $[27,28]$, where the fundamental dielectric dispersion in Rs, dRs, $\mathrm{RbHSO}_{4}$, and $\mathrm{RbH}_{0.3} \mathrm{D}_{0.7} \mathrm{SO}_{4}$ was thoroughly examined, both theoretically and experimentally, and the description of the experiment was much improved, especially for $\mathrm{RbHSO}_{4}$. Recently a new approach to the fitting procedure for $\mathrm{RbHSO}_{4}$ type crystals was applied [22]. This approach was developed in [29-31] for the description of the $\mathrm{KH}_{2} \mathrm{PO}_{4}$ family ferroelectrics. It was shown that at choosing the values of the theory parameters it is crucial to obtain a good fit to the experimental data for the contribution of the ordering subsystem to the specific heat. Following this method, for the $\mathrm{RbHSO}_{4}$ and $\mathrm{RbH}_{0.3} \mathrm{D}_{0.7} \mathrm{SO}_{4}$ a good description of all calculated characteristics was obtained [22].

However, for Rs some qualitative discrepancies between theory and experiment persisted. Thus, it was impossible to simultaneously fit the data for the spontaneous polarization and static dielectric susceptibility; the temperature curves of polarization relaxation time and dynamic dielectric permittivity in the vicinity of the phase transitions in Rs and dRs were qualitatively different from the experimental ones. Method of fitting to the specific heat is useless here, because the specific heat peculiarities at the Curie points in Rs crystals are very small, and no reliable experimental data exist.

It should be noted (see also [32-34]) that the physical characteristics of Rs, $\mathrm{RbHSO}_{4}$, and $\mathrm{NH}_{4} \mathrm{HSO}_{4}$ can be essentially influenced by tunneling of the ordering structure units $[14,15,17,35]$ and by their interaction with phonons [36-38]. Let us note that in $[17,35]$ the isotopic effect in $\operatorname{Rs}_{1-x} \mathrm{dRs}_{x}$ was attributed to the changes in tunneling only. In [38] a generalized pseudospin-phonon model of a partially deuterated order-disorder type ferroelectrics with asymmetric double-well potential was proposed and studied. Using a decoupling procedure for the Green's functions, within which the pseudospin-phonon interaction can be taken into account more consistent- 
ly than within the random phase approximation, the Green's functions, longitudinal dielectric susceptibility, and coupled pseudospin-phonon vibrations of $\mathrm{Rs}_{1-x} \mathrm{dR}_{x}$ and $\mathrm{N}\left(\mathrm{H}_{1-x} \mathrm{D}_{x}\right)_{4} \mathrm{H}_{1-x} \mathrm{D}_{x} \mathrm{SO}_{4}$ ferroelectrics were calculated. Thermodynamic characteristics of the systems were calculated within the mean field appoximation. No numerical analysis of the results obtained in [38] was performed.

The origin of the remaining qualitative discrepancies between theory and experiment for Rs and dRs was still unclear. Could it be that the MFA is a too poor approximation? The presence of chain fragments of ordering structure elements most likely indicated the necessity of using a better approximation than the MFA. In $[39,40]$ a model of two chains of Ising spins moving in an asymmetric double well potential was proposed; an interaction between the chains is taken into account within the MFA, whereas the interactions between nearest neighbors within the chains are taken into account exactly. In [41] the approach was further improved by exact accounting for the interactions between the pair of chains. These improvements were shown to "deform" the phase diagram (see [14,21]); temperature curve of polarization at different values of the model parameters was explored.

Thus, the task to calculate the physical characteristics of the Mitsui model within the two-particle cluster approximation appeared quite natural. It was fulfulled in [42], where an original approach to the description of thermodynamic and dynamic characteristics of mixed order-disorder type ferroelectrics with asymmetric double-well potential was proposed. Within the two-particle cluster approximation for the short-range interactions and mean field approximation for the long-range interactions, the thermodynamic potentials, static and dynamic Green's functions for annealed and quenched systems were calculated. The obtained expressions for the thermodynamic and dynamic characteristics of $\mathrm{Rs}_{1-x} \mathrm{dRs}_{x}, \mathrm{Rb}\left(\mathrm{H}_{1-x} \mathrm{D}_{x}\right) \mathrm{SO}_{4}$, and $\mathrm{N}\left(\mathrm{H}_{1-x} \mathrm{D}_{x}\right){ }_{4} \mathrm{H}_{1-x} \mathrm{D}_{x} \mathrm{SO}_{4}$ crystals contained an increased number of the fitting parameters, which could potentially improve a description of experimental data, in particular for Rs, dRs, $\mathrm{RbHSO}_{4}$. However, this has not removed the above mentioned qualitative and quantitative discrepancies between theory and experiment for Rs and dRs.

This problem was recently solved by considering the piezoelectric properties of Rs crystals. In [43-45] we modified the conventional Mitsui model for Rs by taking into account the piezoelectric coupling between the ordering structure elements and the shear strain $\varepsilon_{4}$. It permitted us to calculate and obtain a good description of experimental data for the elastic and piezoelectric characteristics of Rochelle salt, to obtain dielectric permittivities of free and clamped crystals, and to properly describe the temperature curves of relaxation times and dynamic dielectric permittivities near the Curie points. In [45] the influence of shear stress $\sigma_{4}$ on the physical characteristics of Rochelle salt was studied.

In the present work we explore the temperature and composition dependences of thermodynamic, dielectric, elastic, and piezoelectric characteristics of the $\mathrm{Rs}_{1-x} \mathrm{dRs}_{x}$ crystals. A disordered Mitsui model is considered with taking into account the piezoelectric coupling between the ordering structure units and shear strain $\varepsilon_{4}$. Calculations for the deuterated Rs within the model modified by piezoelectric effects have been also presented for the first time. 


\section{Thermodynamics of the system}

Let us consider the behavior of a disordered (partially deuterated) piezoelectric Rochelle salt crystal. So far it is unclear which structure elements of Rochelle salt lattice play the role of ordering units in a phase transition. However, since there is a certain (though rather weak) isotopic effect for the transition temperatures in these crystals, we may assume that the order-disorder motion in the system somehow involves the motion of hydrogens (protons or deuterons). We suppose that in mixed (partially deuterated) crystals there are two interpenetrating subsystems of ordering structure elements: one is associated with protons and the other with deuterons.

Calculations are performed within the Mitsui model with taking into account the piezoelectric coupling. Then, we should introduce different constants for pair interactions between the structure ordering units: 1) when both units are associated with protons; 2) with deuterons; 3) when one unit is associated with a proton, while the other with a deuteron. The model Hamiltonian then reads

$$
\begin{aligned}
H= & \frac{N}{2} v c_{44}^{E 0} \varepsilon_{4}^{2}-N v e_{14}^{0} E_{1}-\frac{N}{2} v \chi_{11}^{\varepsilon 0} E_{1}^{2}-\frac{1}{2} \sum_{\substack{q f \alpha \\
q^{\prime} f^{\prime} \beta}} R_{q q^{\prime}}\left(\begin{array}{c}
\alpha \beta \\
f f^{\prime}
\end{array}\right) S_{q f}^{z}(\alpha) S_{q^{\prime} f^{\prime}}^{z}(\beta) \\
& -\sum_{q f \alpha}\left[\Delta_{f \alpha}-\left(\mu_{\alpha} E_{1}-2 \psi_{4 \alpha} \varepsilon_{4}\right)\right] S_{q f}^{z}(\alpha) .
\end{aligned}
$$

Three first terms in (2.1) correspond to that part of elastic, piezoelectric, and electric energies, which is attributed to the heavy ions lattice and independent of the arrangement of the ordering units $\left(c_{44}^{E 0}, e_{14}^{0}, \chi_{11}^{\varepsilon 0}\right.$ are the "seed" elastic constant, coefficient of piezoelectric stress, and dielectric susceptibility, respectively); $v$ is the unit cell volume. The lattice strain $\varepsilon_{4}$ and the "seed" constants are assumed to be composition dependent and averaged over the crystal.

The fourth term describes a direct interaction between the ordering structure units; $R_{q q^{\prime}}\left(\begin{array}{c}\alpha \beta \\ 11\end{array}\right)=R_{q q^{\prime}}\left(\begin{array}{c}\alpha \beta \\ 22\end{array}\right)=J_{q q^{\prime}}(\alpha \beta)$ and $R_{q q^{\prime}}\left(\begin{array}{c}\alpha \beta \\ 12\end{array}\right)=R_{q q^{\prime}}\left(\begin{array}{c}\alpha \beta \\ 21\end{array}\right)=K_{q q^{\prime}}(\alpha \beta)$ are the potentials of interaction between the ordering units belonging to the same and to different sublattices, respectively; indices $f, f^{\prime}=1,2$ number the sublattices, whereas $\alpha, \beta=p, d$ correspond to subsystems of ordering units associated with protons $(p)$ and deuterons $(d)$. The fifth term is the energy associated with the assymetry of the potential profile $\left(\Delta_{1 \alpha}=-\Delta_{2 \alpha}=\Delta_{\alpha}\right)$. The sixth and seventh terms are the interaction of the ordering structure elements with external electric field and internal field created by the piezoelectric coupling [43], $\mu_{\alpha}$ is the effective electric moment per unit cell.

The operator of the internal degrees of freedom $S_{q f}^{z}(\alpha)$ by which the state of the ordering structure elements is described can be written in the following form

$$
S_{q f}^{z}(\alpha)=X_{q f}^{\alpha \alpha} S_{q f}^{z}
$$

where

$$
X_{q f}^{p p}=\left(\begin{array}{cc}
1 & 0 \\
0 & 0
\end{array}\right), \quad X_{q f}^{d d}=\left(\begin{array}{cc}
0 & 0 \\
0 & 1
\end{array}\right), \quad X_{q f}^{p p}+X_{q f}^{d d}=1
$$


are the Hubbard operatores, obeying the following permutation relations

$$
\left[X_{q f}^{\alpha \beta} X_{q^{\prime} f^{\prime}}^{\alpha^{\prime} \beta^{\prime}}\right]=\left[X_{q f}^{\alpha \beta^{\prime}} \delta_{\beta \alpha^{\prime}}-X_{q f}^{\alpha^{\prime} \beta} \delta_{\beta^{\prime} \alpha}\right] \delta_{f f^{\prime}} \delta_{q q^{\prime}}
$$

Hereafter, the thermodynamic and dynamic characteristics of the considered ferroelectric systems will be calculated within the mean field approximation. After an identity transformation of the quasispin operators

$$
S_{q f}^{z}(\alpha)=\left\langle S_{q f}^{z}(\alpha)\right\rangle+\left[S_{q f}^{z}(\alpha)-\left\langle S_{q f}^{z}(\alpha)\right\rangle\right] \equiv \frac{1}{2} \bar{\eta}_{f}(\alpha)+\Delta S_{q f}^{z}(\alpha),
$$

the initial Hamiltonian (2.1) can be presented as

$$
\hat{H}=U+\hat{H}^{\prime}+\hat{H}_{0}
$$

where

$$
\begin{aligned}
U & =\frac{1}{8} \sum_{\substack{q, f, \alpha \\
q^{\prime} f^{\prime} \beta}} R_{q q^{\prime}}\left(\begin{array}{c}
\alpha \beta \\
f f^{\prime}
\end{array}\right) \bar{\eta}_{f}(\alpha) \bar{\eta}_{f^{\prime}}(\beta)+\frac{N}{2} v c_{44}^{E 0} \varepsilon_{4}^{2}-N v e_{14}^{0} \varepsilon_{4} E_{1}-\frac{N}{2} v \chi_{11}^{\varepsilon 0} E_{1}^{2}, \\
\hat{H}^{\prime} & =-\frac{1}{2} \sum_{\substack{q, f, \alpha \\
q^{\prime} f^{\prime} \beta}} R_{q q^{\prime}}\left(\begin{array}{c}
\alpha \beta \\
f f^{\prime}
\end{array}\right) \Delta S_{q f}^{z}(\alpha) \Delta S_{q^{\prime} f^{\prime}}^{z}(\beta), \\
\hat{H}_{0} & =-\sum_{q f \alpha} \bar{\varepsilon}_{f}(\alpha) S_{q f}^{z}(\alpha),
\end{aligned}
$$

and $\bar{\varepsilon}_{f}(\alpha)$ is the local field acting on the quasispins associated with protons $(p)$ or deuterons $(d)$ in the $f$ th sublattice

$$
\begin{aligned}
& \bar{\varepsilon}_{1}(p)=\frac{1}{2} \sum_{\beta} J_{0}(p \beta) \bar{\eta}_{1}(\beta)+\frac{1}{2} \sum_{\beta} K_{0}(p \beta) \bar{\eta}_{2}(\beta)+\Delta_{p}-2 \psi_{4 p} \varepsilon_{4}+\mu_{p} E_{1} \\
& \bar{\varepsilon}_{1}(d)=\frac{1}{2} \sum_{\alpha} J_{0}(\alpha d) \bar{\eta}_{1}(\alpha)+\frac{1}{2} \sum_{\alpha} K_{0}(\alpha d) \bar{\eta}_{2}(\alpha)+\Delta_{d}-2 \psi_{4 d} \varepsilon_{4}+\mu_{d} E_{1} \\
& \bar{\varepsilon}_{2}(p)=\frac{1}{2} \sum_{\beta} J_{0}(p \beta) \bar{\eta}_{2}(\beta)+\frac{1}{2} \sum_{\beta} K_{0}(p \beta) \bar{\eta}_{1}(\beta)-\Delta_{p}-2 \psi_{4 p} \varepsilon_{4}+\mu_{p} E_{1}, \\
& \bar{\varepsilon}_{2}(d)=\frac{1}{2} \sum_{\alpha} J_{0}(\alpha d) \bar{\eta}_{2}(\alpha)+\frac{1}{2} \sum_{\alpha} K_{0}(\alpha d) \bar{\eta}_{1}(\alpha)-\Delta_{d}-2 \psi_{4 d} \varepsilon_{4}+\mu_{d} E_{1} .
\end{aligned}
$$

In further calculations the term $\hat{H}^{\prime}$ in the Hamiltonian will be neglected.

To obtain the observable quantities, we should perform both thermodynamical and configurational (over sort configurations) averagings. We consider a case of quenched disorder, when distribution of quasispins associated with protons or deuterons over the lattice is fixed and temperature independent. Therefore, the thermodynamical averaging refers to the spin degrees of freedom only. For instance, for the single-particle distribution function one has

$$
\bar{\eta}_{f}(\alpha)=\left\langle\frac{\operatorname{Sp} S_{q f}^{z}(\alpha) \mathrm{e}^{-\beta \hat{H}_{0}}}{\mathrm{Sp} \mathrm{e}^{-\beta \hat{H}_{0}}}\right\rangle_{x}=\left\langle X_{q f}^{\alpha \alpha} \tanh \frac{1}{2} \beta \bar{\varepsilon}_{f}(\alpha)\right\rangle_{x}=x_{\alpha} \tanh \frac{1}{2} \beta \bar{\varepsilon}_{f}(\alpha)
$$


( $x_{\alpha}$ is the concentration of the $\alpha$ - component).

Let us introduce new variables $\bar{\xi}(\alpha)$ and $\bar{\sigma}(\alpha)$ :

$$
\begin{array}{ll}
\bar{\xi}(p)=\frac{1}{2}\left[\bar{\eta}_{1}(p)+\bar{\eta}_{2}(p)\right], & \bar{\xi}(d)=\frac{1}{2}\left[\bar{\eta}_{1}(d)+\bar{\eta}_{2}(d)\right], \\
\bar{\sigma}(p)=\frac{1}{2}\left[\bar{\eta}_{1}(p)-\bar{\eta}_{2}(p)\right], & \bar{\sigma}(d)=\frac{1}{2}\left[\bar{\eta}_{1}(d)-\bar{\eta}_{2}(d)\right] .
\end{array}
$$

Then the system (2.4) can be presented as

$$
\begin{aligned}
& \bar{\xi}(p)=\frac{1}{2} x_{p}\left\{\tanh \frac{1}{2}\left[\gamma_{1}+\delta_{1}\right]+\tanh \frac{1}{2}\left[\gamma_{1}-\delta_{1}\right]\right\}=x_{p} \frac{\sinh \gamma_{1}}{\cosh \gamma_{1}+\cosh \delta_{1}}, \\
& \bar{\xi}(d)=\frac{1}{2} x_{d}\left\{\tanh \frac{1}{2}\left[\gamma_{2}+\delta_{2}\right]+\tanh \frac{1}{2}\left[\gamma_{2}-\delta_{2}\right]\right\}=x_{d} \frac{\sinh \gamma_{2}}{\cosh \gamma_{2}+\cosh \delta_{2}}, \\
& \bar{\sigma}(p)=\frac{1}{2} x_{p}\left\{\tanh \frac{1}{2}\left[\gamma_{1}+\delta_{1}\right]-\tanh \frac{1}{2}\left[\gamma_{1}-\delta_{1}\right]\right\}=x_{p} \frac{\sinh \delta_{1}}{\cosh \gamma_{1}+\cosh \delta_{1}}, \\
& \bar{\sigma}(d)=\frac{1}{2} x_{d}\left\{\tanh \frac{1}{2}\left[\gamma_{2}+\delta_{2}\right]-\tanh \frac{1}{2}\left[\gamma_{2}-\delta_{2}\right]\right\}=x_{d} \frac{\sinh \delta_{2}}{\cosh \gamma_{2}+\cosh \delta_{2}},
\end{aligned}
$$

where

$$
\begin{aligned}
\gamma_{1} & =\frac{\tilde{R}^{+}(p p)}{2 T} \bar{\xi}(p)+\frac{\tilde{R}^{+}(p d)}{2 T} \bar{\xi}(d)-\frac{2}{T} \tilde{\psi}_{4 p} \varepsilon_{4}+\frac{\mu_{p} E_{1}}{k T} \\
\delta_{1} & =-\frac{\tilde{R}^{-}(p p)}{2 T} \bar{\sigma}(p)-\frac{\tilde{R}^{-}(p d)}{2 T} \bar{\sigma}(d)+\frac{\tilde{\Delta}_{p}}{T} \\
\gamma_{2} & =\frac{\tilde{R}^{+}(p d)}{2 T} \bar{\xi}(p)+\frac{\tilde{R}^{+}(d d)}{2 T} \bar{\xi}(d)-\frac{2}{T} \tilde{\psi}_{4 d} \varepsilon_{4}+\frac{\mu_{d} E_{1}}{k T} \\
\delta_{2} & =-\frac{\tilde{R}^{-}(p d)}{2 T} \bar{\sigma}(p)-\frac{\tilde{R}^{-}(d d)}{2 T} \bar{\sigma}(d)+\frac{\tilde{\Delta}_{d}}{T},
\end{aligned}
$$

and

$$
\tilde{R}^{ \pm}(\alpha \beta)=\frac{1}{k_{\mathrm{B}}}\left[K_{0}(\alpha \beta) \pm J_{0}(\alpha \beta)\right]
$$

To calculate piezoelectric, elastic, dielectric characteristics of the mixed Rochelle salt crystals we shall use the thermodynamic potential (calculated per one pair of quasispins)

$$
\begin{aligned}
g_{1 E}(4) & =\frac{G_{1 E}(4)}{N k_{\mathrm{B}}}= \\
= & -\bar{v} \sigma_{4} \varepsilon_{4}+\frac{1}{2} \bar{v} c_{44}^{E 0} \varepsilon_{4}^{2}-\bar{v} e_{14}^{0} \varepsilon_{4} E_{1}-\frac{1}{2} \bar{v} \chi_{11}^{\varepsilon 0} E_{1}^{2} \\
& +\frac{1}{4}\left(\tilde{R}^{+}(p p) x_{p}^{2} \xi^{2}(p)+\tilde{R}^{+}(d d) x_{d}^{2} \xi^{2}(d)-\tilde{R}^{-}(p p) x_{p}^{2} \sigma^{2}(p)-\tilde{R}^{-}(d d) x_{d}^{2} \sigma^{2}(d)\right) \\
& +\frac{1}{2} \tilde{R}^{+}(p d) x_{p} x_{d} \xi(p) \xi(d)-\frac{1}{2} \tilde{R}^{-}(p d) x_{p} x_{d} \sigma(p) \sigma(d)-2\left(x_{p}+x_{d}\right) T \ln 2 \\
& -T x_{p} \ln \left(\cosh \frac{\gamma_{1}+\delta_{1}}{2} \cosh \frac{\gamma_{1}-\delta_{1}}{2}\right)-T x_{d} \ln \left(\cosh \frac{\gamma_{2}+\delta_{2}}{2} \cosh \frac{\gamma_{2}-\delta_{2}}{2}\right),
\end{aligned}
$$


$\left(\bar{v}=\frac{v}{k_{\mathrm{B}}}\right)$. From the conditions:

$$
\frac{1}{\bar{v}}\left(\frac{\partial g_{1 E}}{\partial \varepsilon_{4}}\right)_{E_{1}, \sigma_{4}}=0, \quad \frac{1}{\bar{v}}\left(\frac{\partial g_{1 E}}{\partial E_{1}}\right)_{\sigma_{4}}=-P_{1}
$$

we obtain that

$$
\begin{aligned}
& \sigma_{4}=c_{44}^{E 0} \varepsilon_{4}-e_{14}^{0} E_{1}+x_{p} \frac{\tilde{\psi}_{4 p}}{\bar{v}} 2 \xi(p)+x_{d} \frac{\tilde{\psi}_{4 d}}{\bar{v}} 2 \xi(d), \\
& P_{1}=e_{14}^{0} \varepsilon_{4}+\chi_{11}^{\varepsilon 0} E_{1}+\frac{\mu_{p}}{v} x_{p} \xi(p)+\frac{\mu_{d}}{v} x_{d} \xi(d) .
\end{aligned}
$$

Using expressions (2.6) and (2.9), let us calculate the static dielectric susceptibility of mixed Rochell salt type crystal along its $a$-axis for the case of a mechanically clamped system

$$
\begin{aligned}
\chi_{11}^{\varepsilon}(0)= & \left(\frac{\partial P_{1}}{\partial E_{1}}\right)_{\varepsilon_{4}}=\chi_{11}^{\varepsilon 0}+\frac{\mu_{p}}{v} x_{p}\left(\frac{\partial \xi(p)}{\partial E_{1}}\right)_{\varepsilon_{4}}+\frac{\mu_{d}}{v} x_{d}\left(\frac{\partial \xi(d)}{\partial E_{1}}\right)_{\varepsilon_{4}}= \\
= & \chi_{11}^{\varepsilon 0}+\frac{1}{2 T \Delta_{4}}\left\{\bar{v} \frac{\mu_{p}^{2}}{v^{2}} x_{p}\left(a_{0} \Delta_{p 1}+c_{0} \Delta_{p 3}\right)+\bar{v} \frac{\mu_{d}^{2}}{v^{2}} x_{d}\left(b_{0} \Delta_{d 2}+d_{0} \Delta_{d 4}\right)\right. \\
& \left.-\bar{v} \frac{\mu_{p} \mu_{d}}{v^{2}} x_{p}\left(b_{0} \Delta_{p 2}+d_{0} \Delta_{p 4}\right)-\bar{v} \frac{\mu_{p} \mu_{d}}{v^{2}} x_{d}\left(a_{0} \Delta_{d 1}+c_{0} \Delta_{d 3}\right)\right\} .
\end{aligned}
$$

The notations used here are given in Appendix.

Similarly, for the coefficient of the piezoelectric stress we get

$$
\begin{aligned}
e_{14}= & \left(\frac{\partial P_{1}}{\partial \varepsilon_{4}}\right)_{E_{1}}=e_{14}^{0}+\frac{\mu_{p}}{v}\left(\frac{\partial \xi(p)}{\partial \varepsilon_{4}}\right)_{E_{1}}+\frac{\mu_{d}}{v}\left(\frac{\partial \xi(d)}{\partial \varepsilon_{4}}\right)_{E_{1}}= \\
= & e_{14}^{0}-\frac{1}{T \Delta_{4}}\left\{\frac{\mu_{p}}{v} \tilde{\psi}_{4 p} x_{p}\left(a_{0} \Delta_{p 1}+c_{0} \Delta_{p 3}\right)-\frac{\mu_{d}}{v} \tilde{\psi}_{4 p} x_{d}\left(a_{0} \Delta_{d 1}+c_{0} \Delta_{d 3}\right)\right. \\
& \left.+\frac{\mu_{d}}{v} \tilde{\psi}_{4 d} x_{d}\left(b_{0} \Delta_{d 2}+d_{0} \Delta_{d 4}\right)-\frac{\mu_{p}}{v} \tilde{\psi}_{4 d} x_{p}\left(b_{0} \Delta_{p 2}+d_{0} \Delta_{p 4}\right)\right\} .
\end{aligned}
$$

And from (2.8) and (2.6) we obtain an expression for the elastic constant of the system at a constant electric field

$$
\begin{aligned}
c_{44}^{E}= & \left(\frac{\partial \sigma_{4}}{\partial \varepsilon_{4}}\right)_{E_{1}}= \\
= & c_{44}^{E 0}+\frac{4}{\bar{v} T \Delta_{4}}\left\{\tilde{\psi}_{4 p}^{2} x_{p}\left(a_{0} \Delta_{p 1}+c_{0} \Delta_{p 3}\right)+\tilde{\psi}_{4 d}^{2} x_{d}\left(b_{0} \Delta_{d 2}+d_{0} \Delta_{d 4}\right)\right. \\
& \left.-\tilde{\psi}_{4 p} \tilde{\psi}_{4 d} x_{p}\left(b_{0} \Delta_{p 2}+d_{0} \Delta_{p 4}\right)-\tilde{\psi}_{4 p} \tilde{\psi}_{4 d} x_{d}\left(a_{0} \Delta_{d 1}+c_{0} \Delta_{d 3}\right)\right\} .
\end{aligned}
$$

The other piezoelectric $\left(h_{14}, d_{14}, g_{14}\right)$, dielectric $\left(\chi_{11}^{\sigma}\right)$ and elastic $\left(c_{44}^{P}, s_{44}^{E}\right)$ characteristics of disordered Rochelle salt type crystal can be obtained from the above found quantities, using known thermodynamic relations.

To find the specific heat of the system we use the free energy equal to

$$
f(4)=g_{1 E}(4)+\bar{v} P_{1} E_{1}+\bar{v} \sigma_{4} \varepsilon_{4}
$$


Respectively, molar entropy of the crystal associated with its quasispin subsystem is as follows:

$$
\begin{aligned}
S_{4}=- & \frac{R}{2}\left(\frac{\partial f(4)}{\partial T}\right)_{P_{1} \varepsilon_{4}}= \\
= & R\left\{\left(x_{p}+x_{d}\right) \ln 2+\frac{x_{p}}{2} \ln \left(\cosh \frac{1}{2}\left(\gamma_{1}+\delta_{1}\right) \cosh \frac{1}{2}\left(\gamma_{1}-\delta_{1}\right)\right)\right. \\
& +\frac{x_{d}}{2} \ln \left(\cosh \frac{1}{2}\left(\gamma_{2}+\delta_{2}\right) \cosh \frac{1}{2}\left(\gamma_{2}-\delta_{2}\right)\right) \\
& \left.-\frac{x_{p} \gamma_{1} \sinh \gamma_{1}+x_{p} \delta_{1} \sinh \delta_{1}}{2\left(\cosh \gamma_{1}+\cosh \delta_{1}\right)}-\frac{x_{d} \gamma_{2} \sinh \gamma_{2}+x_{d} \delta_{2} \sinh \delta_{2}}{2\left(\cosh \gamma_{2}+\cosh \delta_{2}\right)}\right\},
\end{aligned}
$$

where $R$ is the gas constant. Molar specific heat of the quasispin subsystem is obtained by numerical differentiation of entropy

$$
\Delta C_{4}^{\sigma}=T\left(\frac{\mathrm{d} S_{4}}{\mathrm{~d} T}\right)_{\sigma} .
$$

\section{Relaxation dynamics in partially deuterated Rs}

Dynamic dielectric characteristics of mixed ferroelectrics with the asymmetric double well potential will be considered within the Glauber model [16]. Similarly to $[42,43]$ we obtain a system of equations for the single particle distribution functions of quasispins

$$
\begin{aligned}
-\varphi \frac{\mathrm{d}}{\mathrm{d} t} \bar{\xi}(p) & =\bar{\xi}(p)-\frac{1}{2} x_{p}\left[\tanh \frac{1}{2}\left(\gamma_{1}+\delta_{1}\right)+\tanh \frac{1}{2}\left(\gamma_{1}-\delta_{1}\right)\right], \\
-\varphi \frac{\mathrm{d}}{\mathrm{d} t} \bar{\xi}(d) & =\bar{\xi}(d)-\frac{1}{2} x_{d}\left[\tanh \frac{1}{2}\left(\gamma_{2}+\delta_{2}\right)+\tanh \frac{1}{2}\left(\gamma_{2}-\delta_{2}\right)\right], \\
-\varphi \frac{\mathrm{d}}{\mathrm{d} t} \bar{\sigma}(p) & =\bar{\sigma}(p)-\frac{1}{2} x_{p}\left[\tanh \frac{1}{2}\left(\gamma_{1}+\delta_{1}\right)-\tanh \frac{1}{2}\left(\gamma_{1}-\delta_{1}\right)\right], \\
-\varphi \frac{\mathrm{d}}{\mathrm{d} t} \bar{\sigma}(d) & =\bar{\sigma}(d)-\frac{1}{2} x_{d}\left[\tanh \frac{1}{2}\left(\gamma_{2}+\delta_{2}\right)-\tanh \frac{1}{2}\left(\gamma_{2}-\delta_{2}\right)\right] .
\end{aligned}
$$

The general form of the system (3.1) is quite complicated. Hereafter we shall restrict our consideration to the small deviations from equilibrium. Then $\bar{\xi}(\alpha), \bar{\sigma}(\alpha)$ and the electric field $E_{1}$ can be presented as sums of two terms each

$$
\begin{aligned}
\bar{\xi}(\alpha) & =\bar{\xi}_{0}(\alpha)+\bar{\xi}_{t}(\alpha), \\
\bar{\sigma}(\alpha) & =\bar{\sigma}_{0}(\alpha)+\bar{\sigma}_{t}(\alpha), \\
E_{1} & =E_{10}+E_{1 t} .
\end{aligned}
$$

In the case of these small deviations, the expressions $\tanh \left(\gamma_{i} \pm \delta_{i}\right) / 2$ can be expanded in $\bar{\xi}_{t}(\alpha), \bar{\sigma}_{t}(\alpha), E_{t}$ up to the linear terms in a quite wide temperature 
range. We obtain then systems of equations for the equilibrium functions (coinciding with (2.6)) and for the time-dependent parts

$$
\begin{aligned}
-\varphi \frac{\mathrm{d}}{\mathrm{d} t} \bar{\xi}_{t}(p) & =a_{10} \bar{\xi}_{t}(p)+a_{20} \bar{\xi}_{t}(d)+a_{30} \bar{\sigma}_{t}(p)+a_{40} \bar{\sigma}_{t}(d)-a_{00} \frac{\mu_{p} E_{t}}{2 k T} \\
-\varphi \frac{\mathrm{d}}{\mathrm{d} t} \bar{\xi}_{t}(d) & =b_{10} \bar{\xi}_{t}(p)+b_{20} \bar{\xi}_{t}(d)+b_{30} \bar{\sigma}_{t}(p)+b_{40} \bar{\sigma}_{t}(d)-b_{00} \frac{\mu_{d} E_{t}}{2 k T} \\
-\varphi \frac{\mathrm{d}}{\mathrm{d} t} \bar{\sigma}_{t}(p) & =c_{10} \bar{\xi}_{t}(p)+c_{20} \bar{\xi}_{t}(d)+c_{30} \bar{\sigma}_{t}(p)+c_{40} \bar{\sigma}_{t}(d)-c_{00} \frac{\mu_{p} E_{t}}{2 k T} \\
-\varphi \frac{\mathrm{d}}{\mathrm{d} t} \bar{\sigma}_{t}(d) & =d_{10} \bar{\xi}_{t}(p)+d_{20} \bar{\xi}_{t}(d)+d_{30} \bar{\sigma}_{t}(p)+d_{40} \bar{\sigma}_{t}(d)-d_{00} \frac{\mu_{d} E_{t}}{2 k T}
\end{aligned}
$$

the quantities used here $a_{i 0}, b_{i 0}, c_{i 0}, d_{i 0}(i=0-3)$ are obtained from the given in Appendix $a_{i}, b_{i}, c_{i}, d_{i}(i=0-3)$ by changing $\xi(p) \rightarrow \xi_{0}(p), \xi(d) \rightarrow \xi_{0}(d)$, $\sigma(p) \rightarrow \sigma_{0}(d), \sigma(d) \rightarrow \sigma_{0}(d)$.

In the case $E_{t}=0$ the system of equations (3.3) can be reduced to a single differential equation for $\bar{\xi}_{t}(p)$ :

$$
\frac{\mathrm{d}^{(4)} \bar{\xi}_{t}(p)}{\mathrm{d} t^{4}}+\frac{n_{1}}{\varphi} \frac{\mathrm{d}^{(3)} \bar{\xi}_{t}(p)}{\mathrm{d} t^{3}}+\frac{n_{2}}{\varphi^{2}} \frac{\mathrm{d}^{(2)} \bar{\xi}_{t}(p)}{\mathrm{d} t^{2}}+\frac{n_{3}}{\varphi^{3}} \frac{\mathrm{d} \bar{\xi}_{t}(p)}{\mathrm{d} t}+\frac{n_{4}}{\varphi^{4}} \bar{\xi}_{t}(p)=0 .
$$

Here

$$
\begin{aligned}
& n_{1}=a_{10}+b_{20}+c_{30}+d_{40} \\
& n_{2}=\left|\begin{array}{ll}
a_{10} & a_{20} \\
b_{10} & b_{20}
\end{array}\right|+\left|\begin{array}{ll}
a_{10} & a_{30} \\
c_{10} & c_{30}
\end{array}\right|+\left|\begin{array}{ll}
a_{10} & a_{40} \\
d_{10} & d_{40}
\end{array}\right|+\left|\begin{array}{cc}
b_{20} & b_{30} \\
c_{20} & c_{30}
\end{array}\right|+\left|\begin{array}{cc}
b_{20} & b_{40} \\
d_{20} & d_{40}
\end{array}\right|+\left|\begin{array}{c}
c_{30} c_{40} \\
d_{30} d_{40}
\end{array}\right|, \\
& n_{3}=\left|\begin{array}{lll}
a_{10} & a_{20} & a_{30} \\
b_{10} & b_{20} & b_{30} \\
c_{10} & c_{20} & c_{30}
\end{array}\right|+\left|\begin{array}{lll}
a_{10} & a_{20} & a_{40} \\
b_{10} & b_{20} & b_{40} \\
d_{10} & d_{20} & d_{40}
\end{array}\right|+\left|\begin{array}{ccc}
a_{10} & a_{30} & a_{40} \\
c_{10} & c_{30} & c_{40} \\
d_{10} & d_{30} & d_{40}
\end{array}\right|+\left|\begin{array}{ccc}
b_{20} & b_{30} & b_{40} \\
c_{20} & c_{30} & c_{40} \\
d_{20} & d_{30} & d_{40}
\end{array}\right|, \\
& n_{4}=\left|\begin{array}{llll}
a_{10} & a_{20} & a_{30} & a_{40} \\
b_{10} & b_{20} & b_{30} & b_{40} \\
c_{10} & c_{20} & c_{30} & c_{40} \\
d_{10} & d_{20} & d_{30} & d_{40}
\end{array}\right| .
\end{aligned}
$$

A general solution of (3.4) can be written as

$$
\bar{\xi}_{t}(p)=\sum_{i=1}^{4} C_{i} \exp \left(-\frac{t}{\tau_{i}}\right)
$$

where $C_{i}$ are constant coefficients, and $\tau_{i}$ are the relaxation times

$$
\frac{\tau_{i}^{-}}{\varphi}=-\frac{1}{\tilde{q}}
$$

$\tilde{q}=\varphi q$ are roots of the characteristic equation

$$
q^{4}+\frac{n_{1}}{\varphi} q^{3}+\frac{n_{2}}{\varphi^{2}} q^{2}+\frac{n_{3}}{\varphi^{3}} q+\frac{n_{4}}{\varphi^{4}}=0 .
$$


Dynamic dielectric susceptibility is defined as

$$
\chi_{11}(\omega)=\frac{\mu_{p}}{v} \frac{\mathrm{d} \bar{\xi}_{t}(p)}{\mathrm{d} E_{1 t}}+\frac{\mu_{d}}{v} \frac{\mathrm{d} \bar{\xi}_{t}(d)}{\mathrm{d} E_{1 t}} .
$$

Let us solve the non-uniform system of equations (3.3) with respect to $\bar{\xi}_{t}(p)$ and $\bar{\xi}_{t}(d)$. Substituting these solutions into (3.8) and taking into account relations (3.6) and (3.7), we obtain the dynamic susceptibility

$$
\chi_{11}(\omega)=\chi_{11}^{0}+\frac{(\mathrm{i} \omega)^{3} n_{0}^{(1)}+(\mathrm{i} \omega)^{2} n_{0}^{(2)}+\mathrm{i} \omega n_{0}^{(3)}+n_{0}^{(4)}}{v k_{\mathrm{B}} T} \prod_{j=1}^{4} \frac{\tau_{j}}{\left(1+\mathrm{i} \omega \tau_{j}^{-}\right)},
$$

where

$$
\begin{aligned}
& \varphi n_{0}^{(1)}=\mu_{p}^{2} a_{00}+\mu_{d}^{2} b_{00}, \\
& \varphi^{2} n_{0}^{(2)}=\mu_{p}^{2}\left(b_{20} a_{00}+c_{30} a_{00}+d_{40} a_{00}-a_{30} c_{00}\right) \\
& +\mu_{d}^{2}\left(a_{10} b_{00}+c_{30} b_{00}+d_{40} b_{00}-b_{40} d_{00}\right) \\
& -\mu_{p} \mu_{d}\left(b_{10} a_{00}+a_{20} b_{00}+b_{30} c_{00}-a_{40} d_{00}\right) \text {, } \\
& \varphi^{3} n_{0}^{(3)}=\mu_{p}^{2}\left[\left(b_{20} c_{30}-b_{30} c_{20}+b_{20} d_{40}-b_{40} d_{20}+c_{30} d_{40}-c_{40} d_{30}\right) a_{00}\right. \\
& \left.+\left(a_{20} b_{30}-a_{30} b_{20}-a_{30} d_{40}+a_{40} d_{30}\right) c_{00}\right] \\
& +\mu_{d}^{2}\left[\left(a_{10} c_{30}-a_{30} c_{10}+a_{10} d_{40}-a_{40} d_{10}+c_{30} d_{40}-c_{40} d_{30}\right) b_{00}\right. \\
& \left.+\left(b_{30} c_{40}-b_{40} c_{30}-a_{10} b_{40}+a_{40} b_{10}\right) d_{00}\right] \\
& -\mu_{p} \mu_{d}\left[\left(b_{10} c_{30}-b_{30} c_{10}+b_{10} d_{40}-b_{40} d_{10}\right) a_{00}\right. \\
& +\left(a_{20} c_{30}-a_{30} c_{20}+a_{20} d_{40}-a_{40} d_{20}\right) b_{00} \\
& +\left(a_{10} b_{30}-a_{30} b_{10}+b_{30} d_{40}-b_{40} d_{30}\right) c_{00} \\
& \left.-\left(a_{20} b_{40}-a_{40} b_{20}+a_{30} c_{40}-a_{40} c_{30}\right) d_{00}\right] \text {, } \\
& \varphi^{4} n_{0}^{(4)}=\mu_{p}^{2}\left(\left|\begin{array}{ccc}
b_{20} & b_{30} & b_{40} \\
c_{20} & c_{30} & c_{40} \\
d_{20} & d_{30} & d_{40}
\end{array}\right| a_{00}+\left|\begin{array}{ccc}
a_{20} & a_{30} & a_{40} \\
b_{20} & b_{30} & b_{40} \\
d_{20} & d_{30} & d_{40}
\end{array}\right| c_{00}\right) \\
& +\mu_{d}^{2}\left(\left|\begin{array}{ccc}
a_{10} & a_{30} & a_{40} \\
c_{10} & c_{30} & c_{40} \\
d_{20} & d_{30} & d_{40}
\end{array}\right| b_{00}+\left|\begin{array}{ccc}
a_{10} & a_{30} & a_{40} \\
b_{10} & b_{30} & b_{40} \\
c_{10} & c_{30} & c_{40}
\end{array}\right| d_{00}\right) \\
& -\mu_{p} \mu_{d}\left(\left|\begin{array}{ccc}
b_{10} & b_{30} & b_{40} \\
c_{10} & c_{30} & c_{40} \\
d_{20} & d_{30} & d_{40}
\end{array}\right| a_{00}+\left|\begin{array}{ccc}
a_{20} & a_{30} & a_{40} \\
c_{20} & c_{30} & c_{40} \\
d_{20} & d_{30} & d_{40}
\end{array}\right| b_{00}\right. \\
& \left.+\left|\begin{array}{lll}
a_{10} & a_{30} & a_{40} \\
b_{10} & b_{30} & b_{40} \\
d_{10} & d_{30} & d_{40}
\end{array}\right| c_{00}+\left|\begin{array}{ccc}
a_{20} & a_{30} & a_{40} \\
b_{20} & b_{30} & b_{40} \\
c_{20} & c_{30} & c_{40}
\end{array}\right| d_{00}\right)
\end{aligned}
$$

Expression (3.9) can be presented as a sum of simple fractions

$$
\chi_{11}(\omega)=\frac{\chi_{1}}{1+\mathrm{i} \omega \tau_{1}}+\frac{\chi_{2}}{1+\mathrm{i} \omega \tau_{2}}+\frac{\chi_{3}}{1+\mathrm{i} \omega \tau_{3}}+\frac{\chi_{4}}{1+\mathrm{i} \omega \tau_{4}},
$$


whereas the system of equations for $\chi_{i}$ reads

$$
\left(\begin{array}{llll}
n_{11} & n_{12} & n_{13} & n_{14} \\
n_{21} & n_{22} & n_{23} & n_{24} \\
n_{31} & n_{32} & n_{33} & n_{34} \\
n_{41} & n_{42} & n_{43} & n_{44}
\end{array}\right)\left(\begin{array}{c}
\chi_{1} \\
\chi_{2} \\
\chi_{3} \\
\chi_{4}
\end{array}\right)=\left(\begin{array}{l}
n_{1} \\
n_{2} \\
n_{3} \\
n_{4}
\end{array}\right),
$$

where the following notations are used

$$
\begin{array}{lll}
n_{11}=\tau_{2} \tau_{3} \tau_{4}, & n_{12}=\tau_{1} \tau_{3} \tau_{4}, \\
n_{13}=\tau_{1} \tau_{2} \tau_{4}, & n_{14}=\tau_{1} \tau_{2} \tau_{3}, \\
n_{21}=\tau_{2} \tau_{3}+\tau_{2} \tau_{4}+\tau_{3} \tau_{4}, & n_{22}=\tau_{1} \tau_{3}+\tau_{1} \tau_{4}+\tau_{3} \tau_{4}, \\
n_{23}=\tau_{1} \tau_{2}+\tau_{1} \tau_{4}+\tau_{2} \tau_{4}, & & n_{24}=\tau_{1} \tau_{2}+\tau_{1} \tau_{3}+\tau_{2} \tau_{3}, \\
n_{31}=\tau_{2}+\tau_{3}+\tau_{4}, & n_{32}=\tau_{1}+\tau_{3}+\tau_{4}, \\
n_{33}=\tau_{1}+\tau_{2}+\tau_{3}, & n_{34}=\tau_{1}+\tau_{3}+\tau_{2}, \\
n_{41}=1, \quad n_{42}=1, & n_{43}=1, \quad n_{44}=1, \\
n_{1}=\frac{1}{v_{a} k T} \tau_{1} \tau_{2} \tau_{3} \tau_{4} n_{0}^{(1)}, & n_{2}=\frac{1}{v_{a} k T} \tau_{1} \tau_{2} \tau_{3} \tau_{4} n_{0}^{(2)}, \\
n_{3}=\frac{1}{v_{a} k T} \tau_{1} \tau_{2} \tau_{3} \tau_{4} n_{0}^{(3)}, & n_{4}=\frac{1}{v_{a} k T} \tau_{1} \tau_{2} \tau_{3} \tau_{4} n_{0}^{(4)} .
\end{array}
$$

Dielectric permittivity then is equal to

$$
\varepsilon_{11}(\omega)=1+4 \pi \chi(\omega)=\varepsilon_{11}^{\prime}(\omega)-\mathrm{i} \varepsilon_{11}^{\prime \prime}(\omega)
$$

where

$$
\begin{aligned}
& \varepsilon_{11}^{\prime}(\omega)=\varepsilon_{\infty}+\sum_{i=1}^{4} \frac{4 \pi \chi_{i}}{1+\left(\omega \tau_{i}\right)^{2}} \\
& \varepsilon_{11}^{\prime \prime}(\omega)=\sum_{i=1}^{4} \frac{4 \pi \chi_{i} \omega \tau_{i}}{1+\left(\omega \tau_{i}\right)^{2}}
\end{aligned}
$$

and $\varepsilon_{\infty}=1+4 \pi \chi_{\infty}$ is the high-frequency contribution to the dielectric permittivity.

\section{Numerical analysis}

Within the above proposed theory we can calculate the physical characteristics of mixed Rochelle salt crystals with any deuteration level. The theory parameters that have to be set include the parameters for the limiting cases of pure and completely deuterated crystals $\left(J_{0}(\alpha \alpha), K_{0}(\alpha \alpha), \Delta_{\alpha}, \psi_{4 \alpha}, \mu_{1 \alpha}, \varphi_{\alpha}, c_{44 \alpha}^{E 0}, \chi_{11}^{\varepsilon_{11}} e_{14}^{0}\right)$ as well as the parameters relevant to partially deuterated systems $\left(J_{0}(p d), K_{0}(p d)\right)$. The case of a pure crystal has been considered in [43]. In this paper we shall perform calculations for a completely deuterated Rochelle salt and later will attempt to describe the partially deuterated systems. 


\subsection{Completely deuterated Rochelle salt}

First, let us consider the case of a completely deuterated Rochelle salt $\left(x_{p}=\right.$ $0, x_{d}=1$ ). Fitting procedure for a pure Rochelle salt is described in detail in [43]. Here we follow the same procedure.

For the unit cell volume (per two quasispins) we use the same values as for undeuterated Rs

$$
v=0.5219[1+0.00013(T-190)] \cdot 10^{-21} \mathrm{~cm}^{3} .
$$

Following the method proposed in [43], in order to describe the dielectric, piezoelectric, and relaxational characteristics of Rs - second order derivatives of thermodynamic potential, we need to determine the values of the effective dipole moment $\mu_{1 p}$ by fitting to the values of dynamic permittivity $\varepsilon_{11}^{\varepsilon}\left(T_{c 1}\right)$ and $\varepsilon_{11}^{\varepsilon}\left(T_{c 2}\right)$ (data of [46] are used). It yields $\mu_{1 d}$ as a function slightly decreasing with temperature

$$
\mu_{1 d}=[2.1+0.0066(308-T)] \times 10^{-18} \mathrm{esu} \cdot \mathrm{cm} .
$$

It provides a good fit to the mentioned second derivatives of thermodynamic potential, but a rather poor description of the data for spontaneous polarization (see below). For $\mu_{1 p}(T)$ of pure Rs in [43] we used

$$
\mu_{1 p}=[2.52+0.0066(297-T)] \times 10^{-18} \mathrm{esu} \cdot \mathrm{cm} .
$$

Table 1 contains the used values of the parameters $J, K, \Delta, \psi_{4}$ for pure [43] and completely deuterated Rochelle salt crystals as well as the "seed" quantities, obtained by fitting the theory to experimental data. Results of the fitting for deuterated Rochelle salt are discussed below.

Theoretical dependences of spontaneous polarization $P_{1}$ and spontaneous strain $\varepsilon_{4}$ of $\mathrm{dRs}$ are given in figure 4.1. As one can see, the maximal theoretical value of
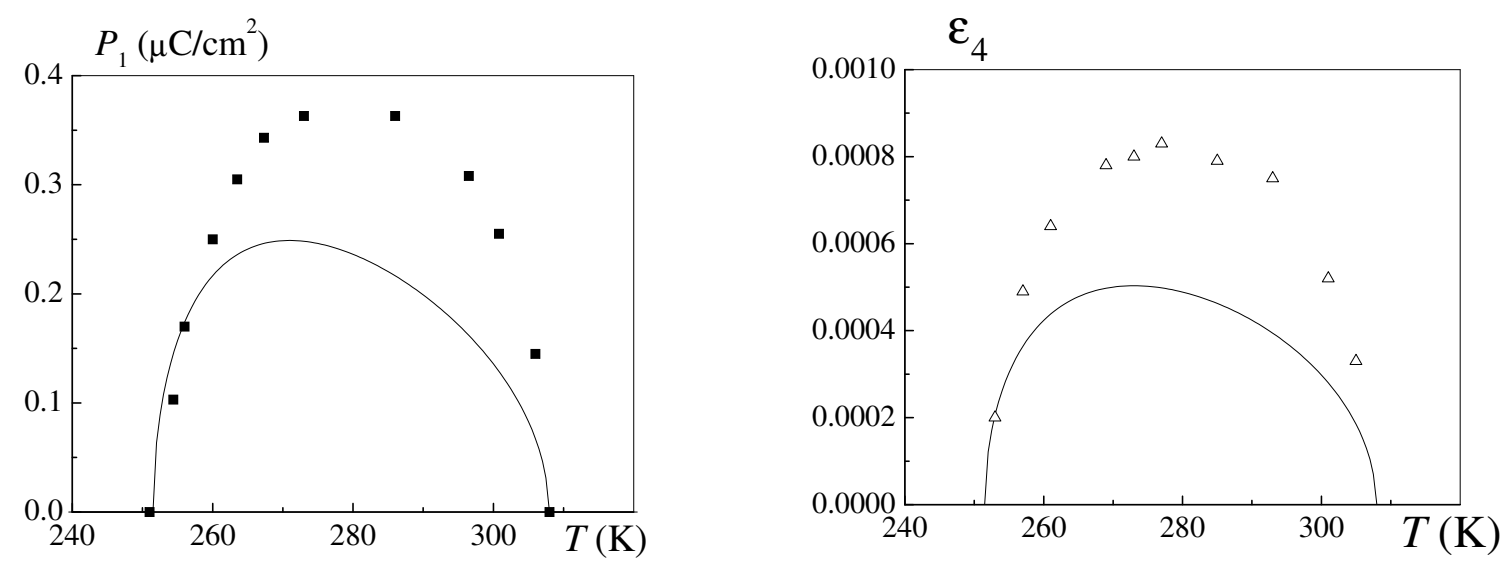

Figure 4.1. Temperature dependences of spontaneous polarization $P_{1}$ and spontaneous strain of dRs at $\sigma_{4}=0 ; \boldsymbol{\square}-[47], \triangle-\varepsilon_{4}=P_{1}\left(\chi_{11}^{\sigma}-\chi_{11}^{\varepsilon}\right) /\left(\chi_{11}^{\sigma} \chi_{11}^{\varepsilon} h_{14}\right)$, where data for $P_{1}$ are taken from [47], for $\chi_{11}^{\sigma}-$ from [47], $\chi_{11}^{\varepsilon}-$ from [46], $h_{14}-$ from [48]. 
Table 4.1. Theory parameters for pure [43] and completely deuterated crystals of Rochelle salt

\begin{tabular}{|c|c|c|c|c|c|c|c|c|}
\hline & $T_{c 1}$, & $T_{c 2}$, & $\tilde{J}_{0}$, & $\tilde{K}_{0}$, & $\begin{array}{c}\tilde{\Delta}, \\
\mathrm{K}\end{array}$ & $\begin{array}{c}\psi_{4}, \\
\mathrm{~K}\end{array}$ & $\begin{array}{c}c_{44}^{E 0}, \\
\mathrm{dyn} / \mathrm{cm}^{2}\end{array}$ & $\begin{array}{c}e_{14}^{0}, \\
\mathrm{esu} / \mathrm{cm}^{2}\end{array}$ \\
\hline $\mathrm{Rs}$ & 255 & 297 & 797.36 & 1468.83 & 737.33 & -760 & $12.8 \times 10^{10}$ & $1 . d 0 \times 10^{4}$ \\
\hline $\mathrm{dRs}$ & 251 & 308 & 806.633 & 1499.532 & 751.861 & -600 & $10.5 \times 10^{10}$ & $0.15 \times 10^{4}$ \\
\hline
\end{tabular}

$P_{1}$ is by $\sim 50 \%$ lower than the experimental one. So far we have no solution to this problem; it could be, however, that there is an error in the rather outdated experimental data. Further meausurements here are thus definitely needed.

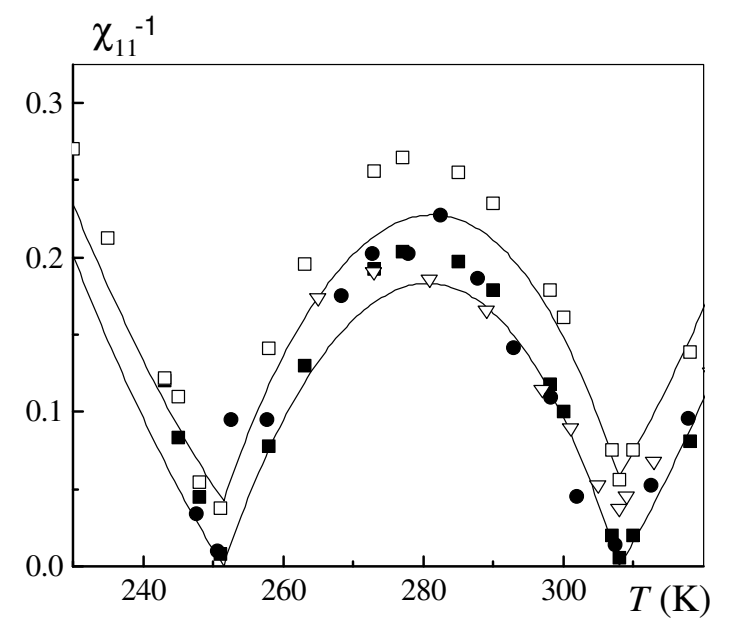

Figure 4.2. Temperature dependence on inverse static dielectric permittivity of free and clamped crystals of dRs. Experimental points are taken from: $-[47]$ $(900 \mathrm{~Hz}), \bullet-[51], \square$ are recalculated from the obtained in [46] Cole-Cole curves, $\nabla-[48]$.

In figure 4.2 we showed the temperature dependences of inverse static dielectric susceptibilities of free $k_{11}^{\sigma}=\left(\chi_{11}^{\sigma}\right)^{-1}$ and clamped $k_{11}^{\varepsilon}=\left(\chi_{11}^{\varepsilon}\right)^{-1}$ crystals of deuterated Rochelle salt. As one can see, the experimental data for $k_{11}^{\varepsilon}(T)$ of [48] and [46] disagree. Experimental values of dielectric permittivity in the lower paraelectric phase should be also verified, since below $240 \mathrm{~K}$ the values of $\varepsilon_{11}^{\sigma}$ [47] become smaller than $\varepsilon_{11}^{\varepsilon}$ obtained from the data of [46]. We get a satisfactory quantitative description of experimental data for $k_{11}^{\sigma}$ of [47] and for $k_{11}^{\varepsilon}$ of [46] both in paraelectric phases and in the ferroelectric phase except for its middle part, where the calculated inverse susceptibilities are smaller than the experimental values.

In figure 4.3 the temperature curves of elastic constants at constant field $c_{44}^{E}$ and at constant polarization $c_{44}^{P}$ of deuterated Rochelle salt are depicted. The elastic constant $c_{44}^{E}$ is essentially temperature dependent, vanishing with the same rate at both Curie points. Theoretical results for $c_{44}^{E}$ are in a good agreement with the data obtained from the formula $c_{44}^{E}=\left(\chi_{11}^{\varepsilon} h_{14}\right)^{2} /\left(\chi_{11}^{\sigma}-\chi_{11}^{\varepsilon}\right)$. The calculated $c_{44}^{P}$ is almost 


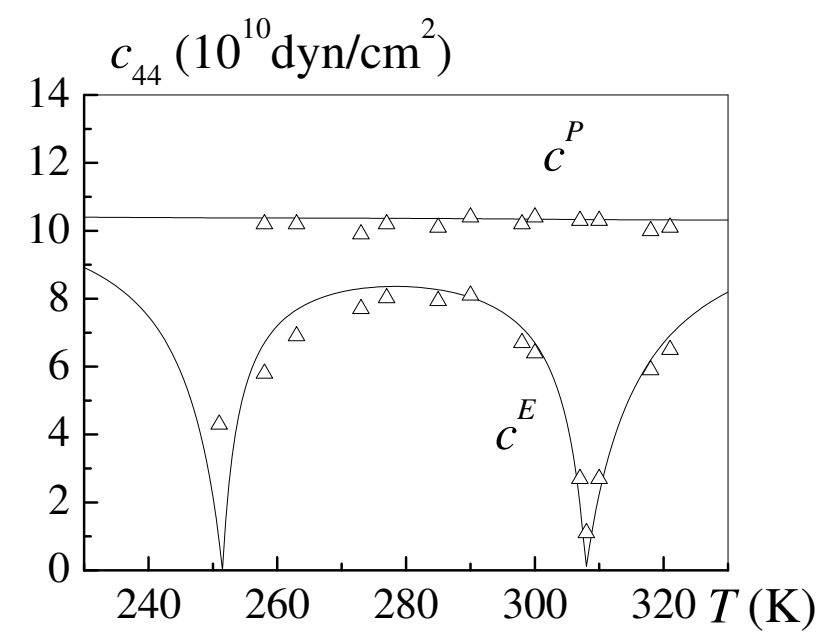

Figure 4.3. Temperature dependence of elastic constants at constant field $c_{44}^{E}$ : $\triangle-c_{44}^{E}=\left(\left(\chi_{11}^{\varepsilon} h_{14}\right)^{2}\right) /\left(\chi_{11}^{\sigma}-\chi_{11}^{\varepsilon}\right)$ and constant polarization $c_{44}^{P}: \triangle-c_{44}^{P}=$ $\left(\chi_{11}^{\sigma} \chi_{11}^{\varepsilon} h_{14}^{2}\right) /\left(\chi_{11}^{\sigma}-\chi_{11}^{\varepsilon}\right)$ of deuterated Rochelle salt crystal. Points for $\chi_{11}^{\sigma}$ are taken from [47], for $\chi_{11}^{\varepsilon}$ from [46], for $h_{14}$ from [48].
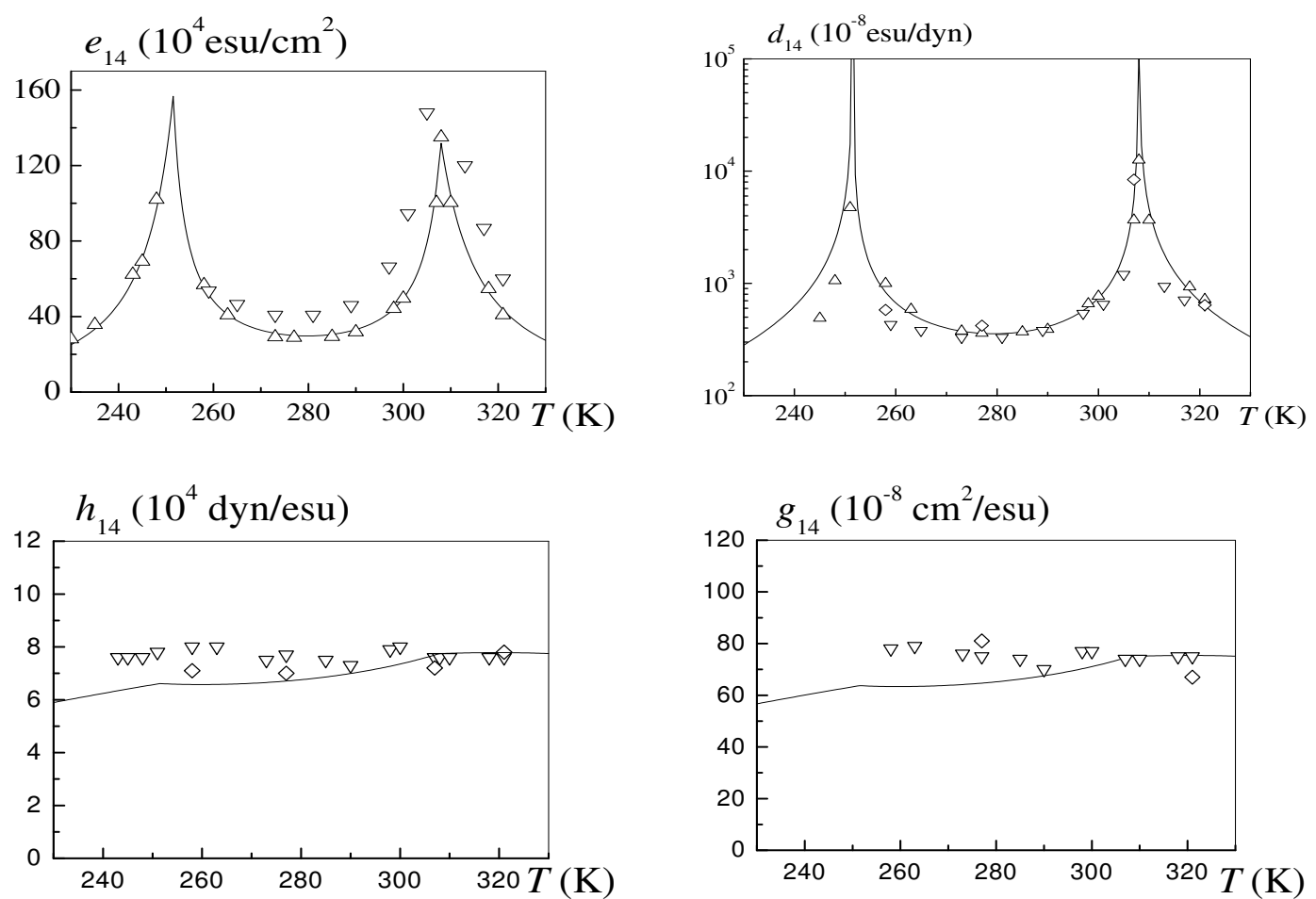

Figure 4.4. Temperature dependences of piezoelectric characteristics of deuterated Rochelle salt. Experimental points are taken from: $\nabla-[48], \diamond-[49], \triangle-$ $d_{14}=\left(\chi_{11}^{\sigma}-\chi_{11}^{\varepsilon}\right) /\left(\chi_{11}^{\varepsilon} h_{14}\right), e_{14}=\chi_{11}^{\varepsilon} h_{14}, g_{14}=\left(\chi_{11}^{\sigma}-\chi_{11}^{\varepsilon}\right) /\left(\chi_{11}^{\sigma} \chi_{11}^{\varepsilon} h_{14}\right)$, values of $\chi_{11}^{\sigma}$ taken from [47], $\chi_{11}^{\varepsilon}$ from [46], $h_{14}$ from [48]. 
temperature independent in all phases and accords with the data obtain from the relation $c_{44}^{P}=\chi_{11}^{\sigma} \chi_{11}^{\varepsilon} h_{14}^{2} /\left(\chi_{11}^{\sigma}-\chi_{11}^{\varepsilon}\right)$.

Figure 4.4 contains theoretical temperature dependences of piezoelectric characteristics of dRs. The obtained curve for $d_{14}(T)$ well agrees with the data of $[48,49]$ as well as with recalculated via the given in caption formula in the entire explored temperature range, except for the low-temperature paraelectric phase.

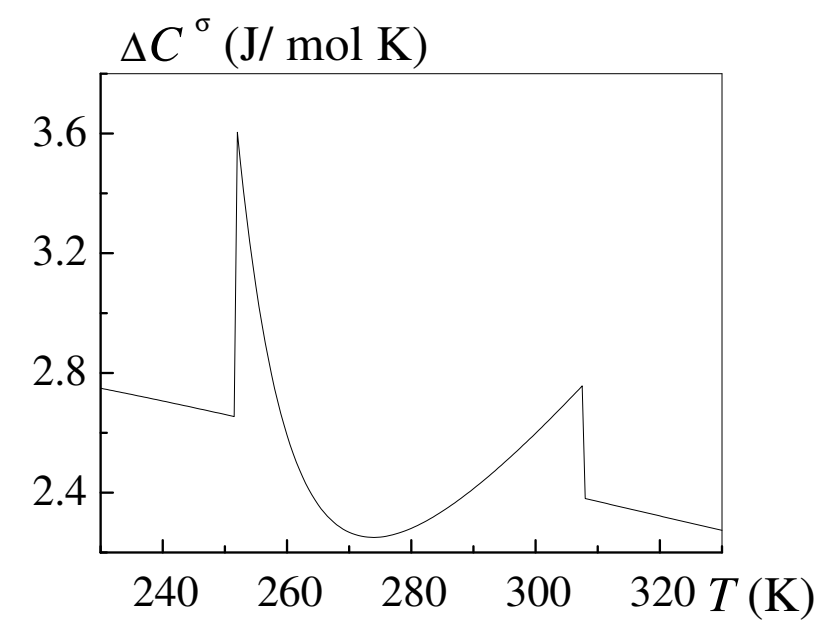

Figure 4.5. Temperature dependence of $\Delta C^{\sigma}$ for deuterated Rochelle salt.

The temperature dependence of the contribution from the ordering units to the specific heat $\Delta C^{\sigma}$ of deuterated Rochelle salt is given in figure 4.5. The theory predicts two positive anomalies of specific heat at both Curie points.

Let us now consider the dielectric relaxation in crystals of dRs. Figure 4.6 contains the calculated temperature dependences of inverse relaxation times $\tau_{1}^{-1}$ and $\tau_{2}^{-1}$, as well as the values of $\tau_{1}^{-1}$ obtained in [46] from experimental data for $\varepsilon_{11}^{*}(\nu, T)$. The latter points are well described by the proposed theory. Peculiar to the tempe-

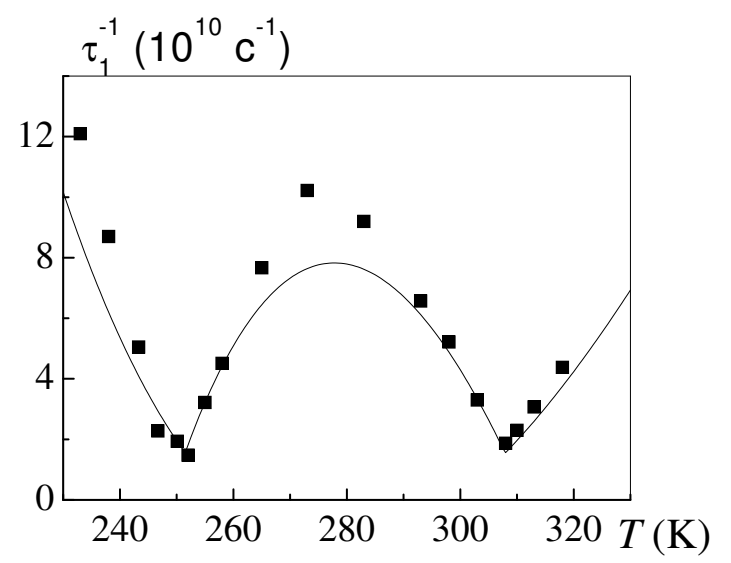

(a)

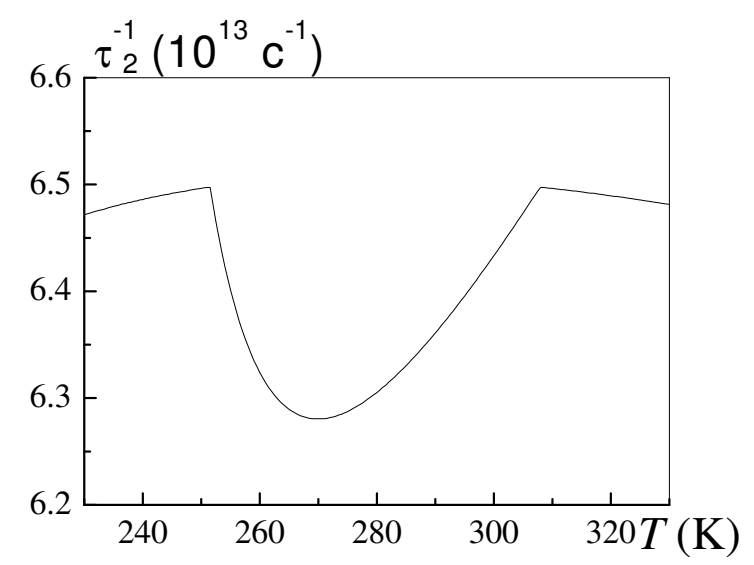

(b)

Figure 4.6. Temperature dependence of inverse relaxation times $\left(\tau_{1}\right)^{-1}$ and $\left(\tau_{2}\right)^{-1}: \mathbf{\square}-[46]$. 
rature curve of $\tau_{1}^{-1}(T)$ is the presence of two finite minima at the transition points. Let us note that any theory for Rochelle salt crystals which does not take into account the piezoelectric effects yields zero values of $\tau_{1}^{-1}(T)$ at these points and, thereby, incorrect temperature dependence of dynamic permittivity in their vicinity. The values of $\tau_{2}$ are three orders smaller than those of $\tau_{1}$.

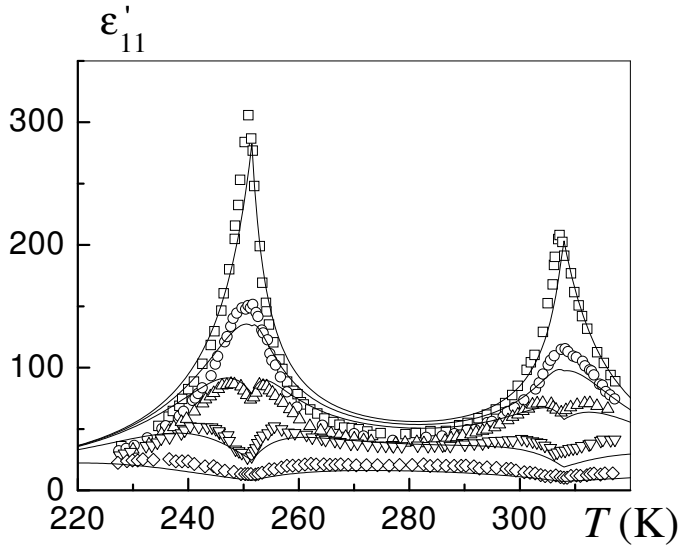

(a)

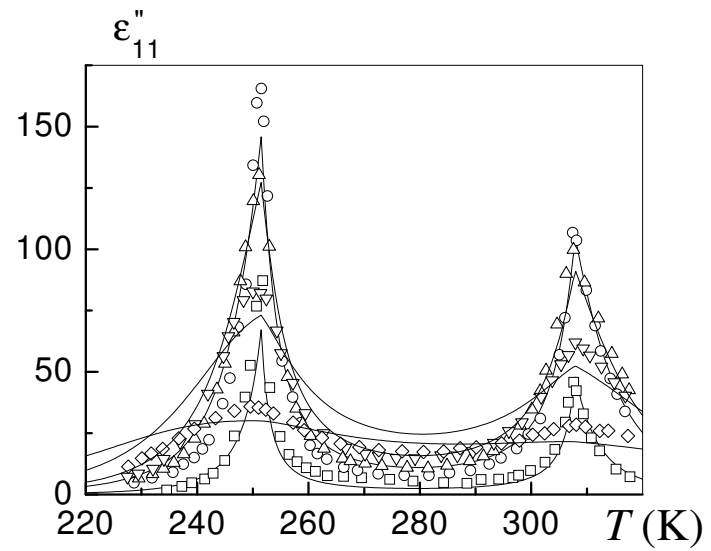

(b)

Figure 4.7. Temperature dependences of real and imaginary parts of dynamic dielectric permittivity $\varepsilon_{11}^{*}$ of deuterated Rochelle salt crystal at different frequencies $\nu(\mathrm{GHz}): \square-0.6 ; \circ-2.8 ; \triangle-4.29 ; \nabla-9.3 ; \diamond-24.0$. Experimental points are taken from $[46]$.

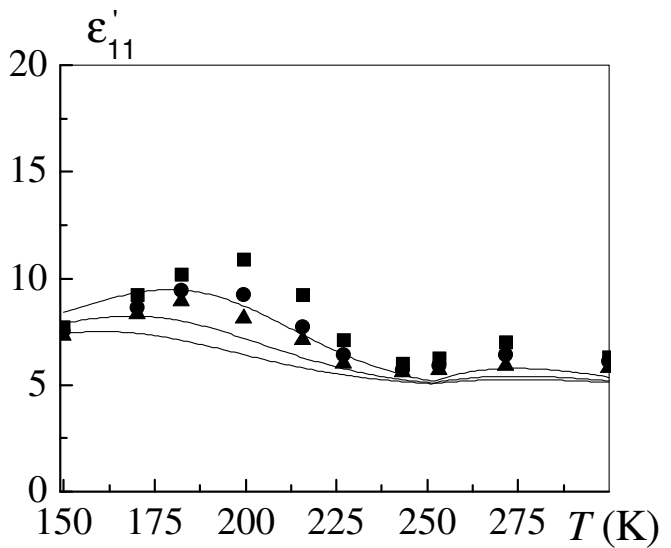

(a)

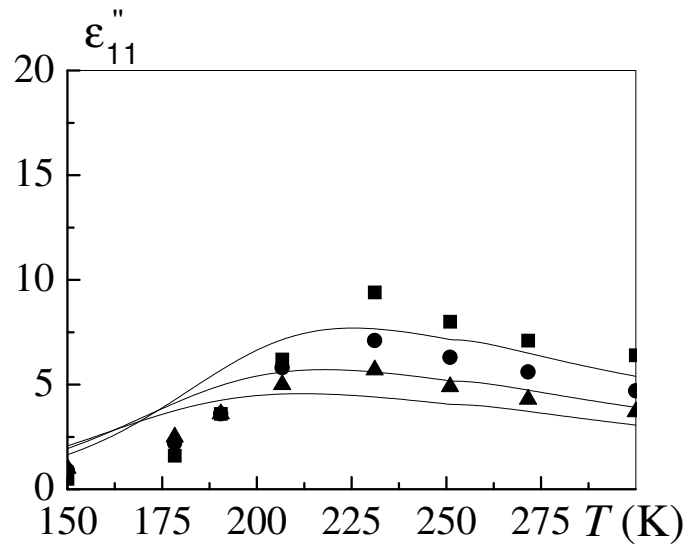

(b)

Figure 4.8. Temperature dependences of real and imaginary parts of dynamic dielectric permittivity $\varepsilon_{11}^{*}$ of deuterated Rochelle salt crystal at different frequencies $\nu(\mathrm{GHz}): \mathbf{\square}-102 ; \bullet-141 ; \boldsymbol{\Delta}-180$. Experimental points are taken from [50].

Temperature dependences of real and imaginary parts of dynamic dielectric permittivity for deuterated Rochelle salt at different frequencies are presented in figure 4.7. Overall, a good description of experimental data [46] is obtained, except for 
$\varepsilon_{11}^{\prime}$ at $0.06,1.5$, and $2.8 \mathrm{GHz}$ in the middle part of the ferroelectric phase as well as for $\varepsilon_{11}^{\prime \prime}$ at $\nu=9.3 \mathrm{GHz}$ at all temperatures studied.

Dynamic permittivity of deuterated Rochelle salt at very high frequencies in the ferroelectric and lower paraelectric phases along with the experimental points of [50] are shown in figure 4.8. As one can see, at these frequencies the theory only qualitatively reproduces the temperature curves for $\varepsilon_{11}^{\prime}$ and $\varepsilon_{11}^{\prime \prime} \quad \varepsilon_{11}^{\prime \prime}(T)$.

Figure 4.9 contains the frequency dependences of dynamic permittivity at different temperatures. As one can see, the agreement between the theory and experiment [46] is particularly good in the upper paraelectric phase.
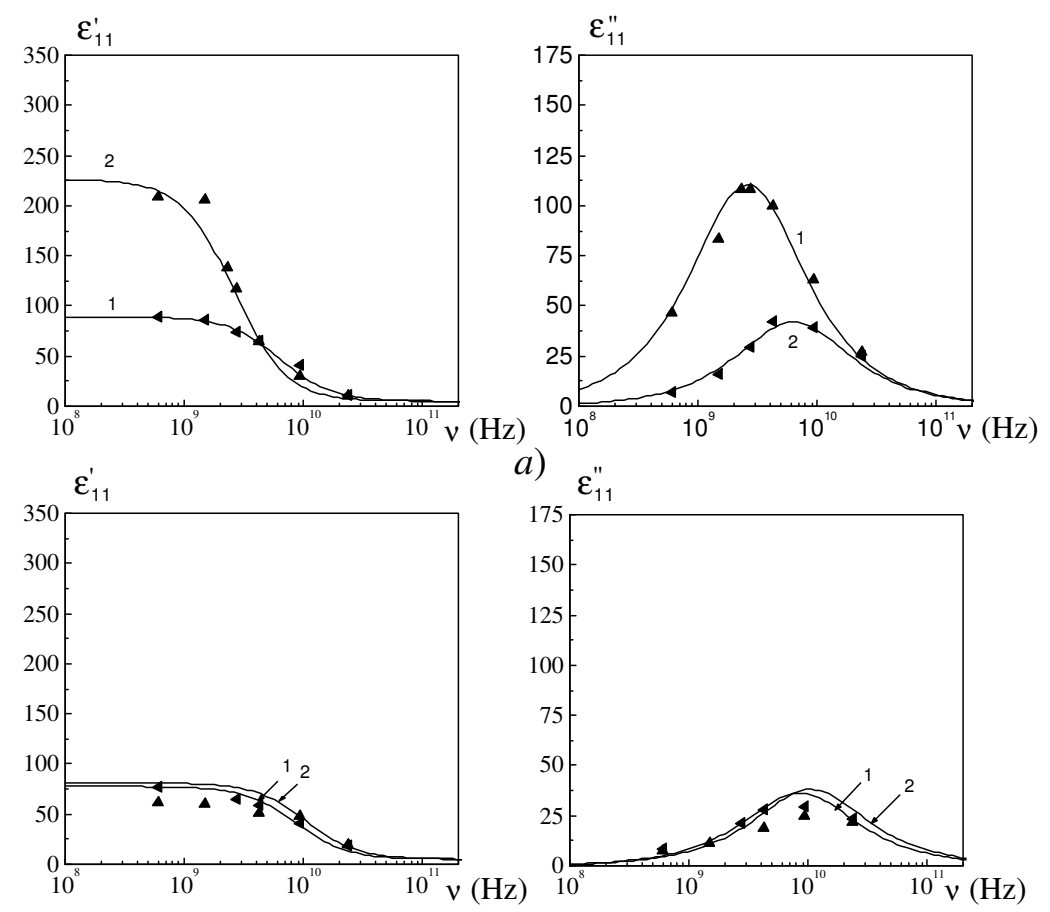

b)
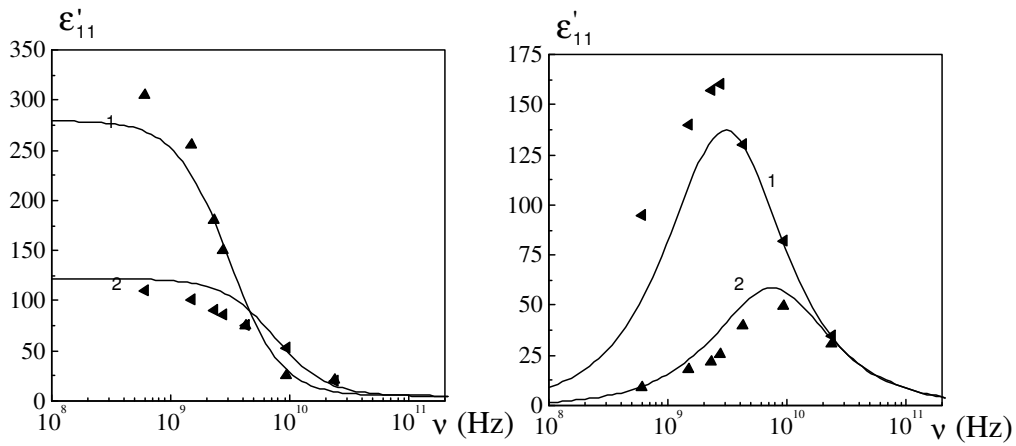

c)

Figure 4.9. Frequency dependences of real and imaginary parts of dynamic dielectric permittivity $\varepsilon_{11}^{*}$ of deuterated Rochelle salt crystal at different temperatures $(\mathrm{K}): \mathrm{a}-308(1), 298(2) ; \mathrm{b}-263(1), 298(2)$; c - 251(1), 243(2). Experimental points are taken from [46]. 

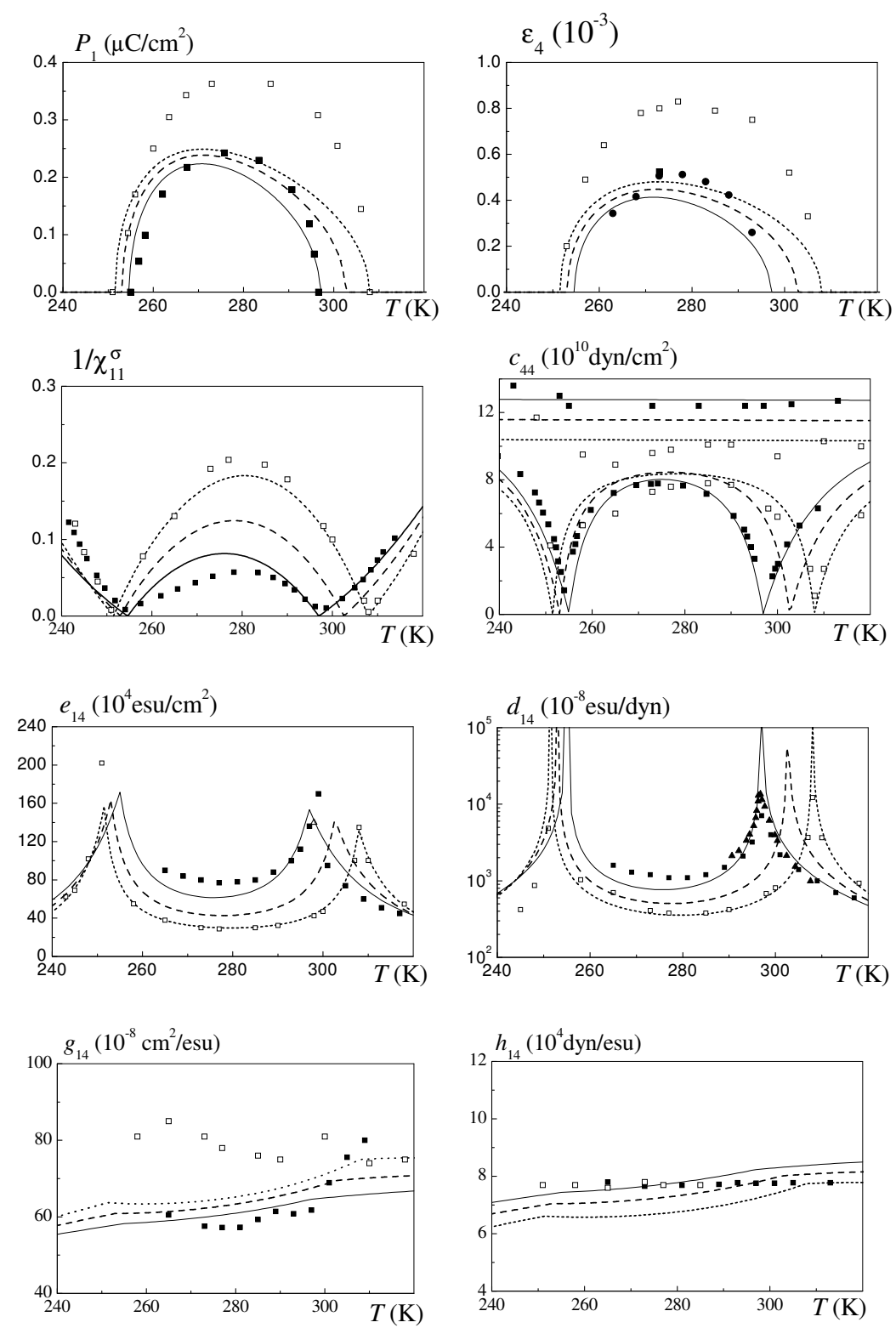

Figure 4.10. Temperature dependences of piezoelectric, dielectric, and elastic characteristics of pure (solid line, solid symbols), partially ( $x=0.5$, dashed line) and completely deuterated (dotted line, open symbols) Rochelle salt. Theory: mean crystal approximation; experimental points are: $P_{1}: \mathbf{\square}, \square-[47]$,

$\varepsilon_{4}: \mathbf{\square}-[55], \bullet-[49], \square-\varepsilon_{4}=P_{1}\left(\chi_{11}^{\sigma}-\chi_{11}^{\varepsilon}\right) /\left(\chi_{11}^{\sigma} \chi_{11}^{\varepsilon} h_{14}\right)$;

$\chi_{11}^{\sigma}: \mathbf{\square}-[56] ; \square-[47] ;$

$c_{44}^{E}: \mathbf{\square}-[52], \square-c_{44}^{E}=\left(\left(\chi_{11}^{\varepsilon} h_{14}\right)^{2}\right) /\left(\chi_{11}^{\sigma}-\chi_{11}^{\varepsilon}\right)$;

$c_{44}^{P}: \mathbf{\square}-[54], \square-c_{44}^{P}=\left(\chi_{11}^{\sigma} \chi_{11}^{\varepsilon} h_{14}^{2}\right) /\left(\chi_{11}^{\sigma}-\chi_{11}^{\varepsilon}\right)$;

$e_{14}: \mathbf{\square}-[53], \square-e_{14}=\chi_{11}^{\varepsilon} h_{14}$;

$d_{14}: \mathbf{\square}-[53], \boldsymbol{\Delta}-[57], \square-d_{14}=\left(\chi_{11}^{\sigma}-\chi_{11}^{\varepsilon}\right) /\left(\chi_{11}^{\varepsilon} h_{14}\right)$,

$h_{14}: \mathbf{\square}-[54], \square-[48]$;

$g_{14}: \boldsymbol{\square}-[53], \square-g_{14}=\left(\chi_{11}^{\sigma}-\chi_{11}^{\varepsilon}\right) /\left(\chi_{11}^{\sigma} \chi_{11}^{\varepsilon} h_{14}\right)$.

Values for $P_{1}, \chi_{11}, h_{14}$ used to calculate the points for dRs are taken from [47] $\left(P_{1}, \chi_{11}^{\sigma}\right),[46]\left(\chi_{11}^{\varepsilon}\right),[48]\left(h_{14}\right)$. 


\subsection{Partially deuterated crystals. Mean crystal appoximation}

Let us now consider the isotopic effect in Rochelle salt crystals. First we shall explore a pure model ([43]; the same formulas are obtained from the ones presented in previous section by putting $x_{p}=0$ or $\left.x_{d}=0\right)$. We use the mean crystal approximation, attributing the isotopic effect to the monotonic changes in interaction parameters. Thus, for a partially deuterated crystal with deuteration level $x=x_{d}$ we take

$$
J=J_{0}(p p)\left(1-x_{d}\right)+J_{0}(d d) x_{d}, K=K_{0}(p p)\left(1-x_{d}\right)+K_{0}(d d) x_{d}, \quad \text { etc, }
$$

where the parameters for pure $(p p)$ and completely deuterated $(d d)$ crystals of Rochelle salt are given in table 1.

Results of such calculations are given in figure 4.10. As one can see, the increase of deuteration leads to the widening of the ferroelectric phase, to the increase of the maximal values of spontaneous polarization $P_{1}$ and strain $\varepsilon_{4}$, and of constant of piezoelectric strain $g_{14}$ and to the decrease of static dielectric susceptibilities of free $\chi_{11}^{\sigma}$ and clamped $\chi_{11}^{\varepsilon}$ crystals in the ferroelectric phase. In the paraelectric phases the slopes of the temperature curves for $\left(\chi_{11}^{\sigma}\right)^{-1}$ and $\left(\chi_{11}^{\varepsilon}\right)^{-1}$ are almost independent of deuteration level.

Maximal value of the elastic constant $c_{44}^{E}$ in the ferroelectric phase is practically the same for Rs and dRs, as well as the rate of changes in $c_{44}^{E}$ with temperature on approaching the Curie points. Elastic constant $c_{44}^{P}$ with the increasing $x$ decreases from $13 \cdot 10^{10} \mathrm{dyn} / \mathrm{cm}^{2}$ for Rs to $10 \cdot 10^{10} \mathrm{dyn} / \mathrm{cm}^{2}$ for $\mathrm{dRs}$.

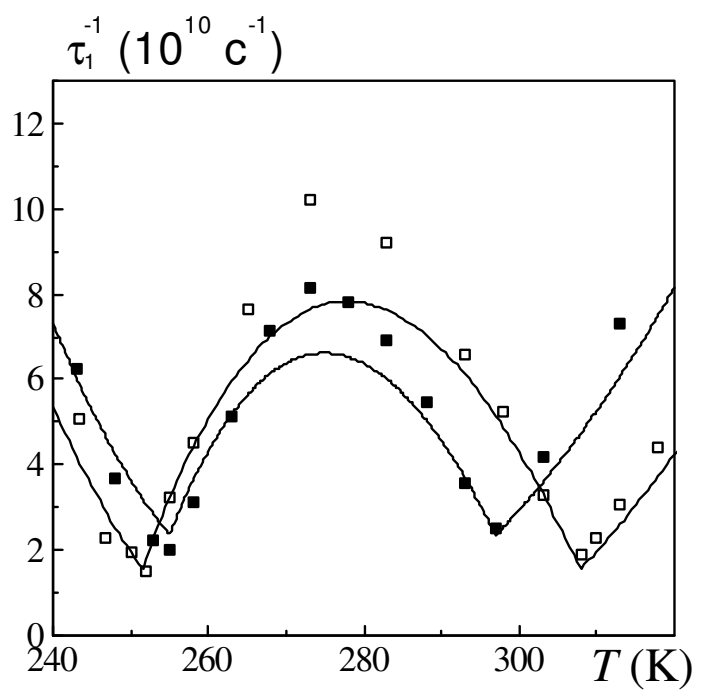

Figure 4.11. Temperature dependence of inverse relaxation time $\tau_{1}^{-1}$ of Rs and dRs crystals. Experimental points are taken from $\square-[46], \mathbf{\square}-[12]$.

Experimental points for the constant of piezoelectric stress $h_{14}$ for Rs and dRs practically coincide at all temperatures, whereas the theoretical values of $h_{14}$ for $\mathrm{dRs}$ are somewhat smaller that for Rs. The theory predicts that on increasing the 
deuteration level, the minimal values of the coefficients of piezoelectric stress $e_{14}$ and strain $d_{14}$ decrease in the ferroelectric phase; the peak values of $e_{14}$ at the transition points decrease as well.

In figure 4.11 the temperature dependences of inverse relaxation time $\left(\tau_{1}\right)^{-1}$ for Rs and dRs are compared. Deuteration decreases with values of $\tau_{1}^{-1}$ at the transition points and in the paraelectric phases. In the middle of the ferroelectric phase, $\tau_{1}^{-1}$ in $\mathrm{Rs}$ is smaller than in dRs.

\subsection{Partially deuterated crystals. A general theory}

Let us now consider a more general case and attempt to describe the deuteration effects in Rochelle salt crystals within the framework of the above developed ("complete") theory. At the absence of experimental data even for transition temperatures of partially deuterated crystals of Rochelle salt, we cannot determine the values of $J_{(\mathrm{pd})}$ and $K_{(\mathrm{pd})}$. Therefore, we shall perform calculations at different trial values of these parameters, in order to find out in what way the changes of these trial values affect the calculated physical characteristics of partially deuterated system.
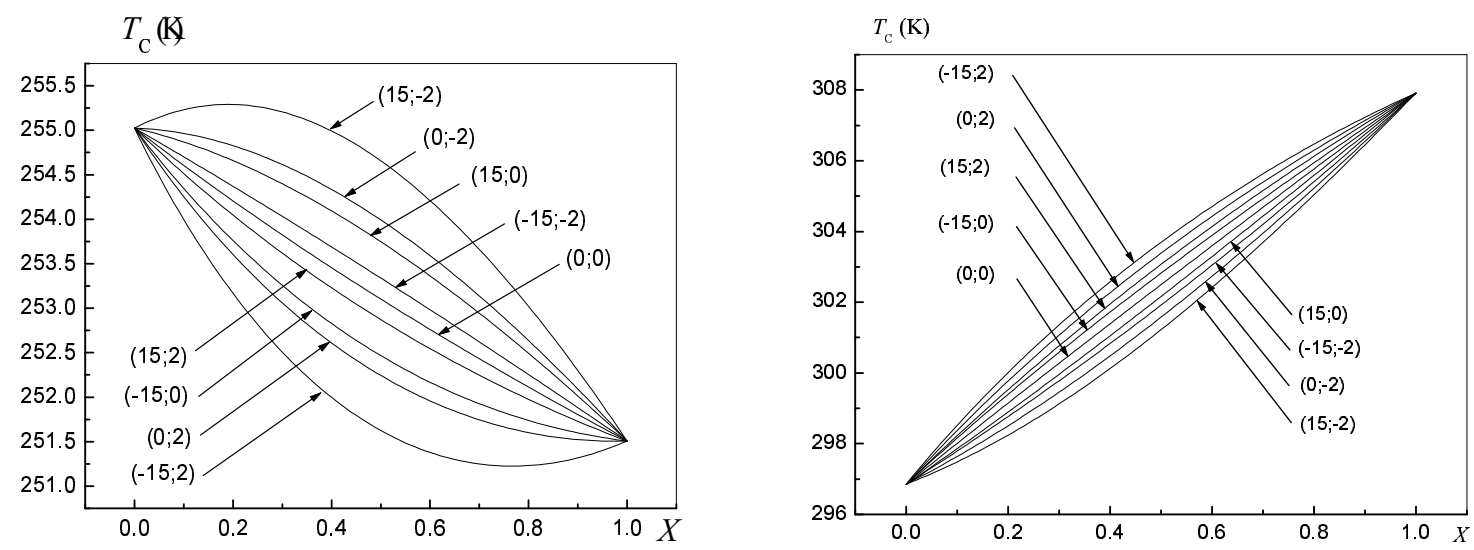

Figure 4.12. Composition dependence $\left(x=x_{d}\right)$ of transition temperatures of mixed Rochelle salt crystals (deuteration levels indicated) calculated within a complete theory.

First, let us define the arithmetic mean set values of the pd-parameters as

$$
\begin{array}{cl}
\tilde{J}_{0}^{\text {arth }}(p d)=\frac{\tilde{J}_{0}(p p)+\tilde{J}_{0}(d d)}{2}, & \tilde{J}_{0}^{\text {arth }}(p d)=802.0115 \mathrm{~K}, \\
\tilde{K}_{0}^{\text {arth }}(p d)=\frac{\tilde{K}_{0}(p p)+\tilde{K}_{0}(d d)}{2}, & \tilde{K}_{0}^{\text {arth }}(p d)=1484.181 \mathrm{~K},
\end{array}
$$

The sets of pd-parameters used in calculations are determined by a relative deviation from the arithmetic mean set of the pd-parameters, denoted by two numbers $(u, v)$.

$$
u=\frac{\tilde{J}_{0}(p d)-\tilde{J}_{0}^{\text {arth }}(p d)}{\left|\tilde{J}_{0}(p p)-\tilde{J}_{0}(d d)\right|} \cdot 100 \%
$$




$$
v=\frac{\tilde{K}_{0}(p d)-\tilde{K}_{0}^{\operatorname{arth}}(p d)}{\left|\tilde{K}_{0}(p p)-\tilde{K}_{0}(d d)\right|} \cdot 100 \%
$$

Thus $(-15 ; 2)$ stands for

$$
\begin{aligned}
& \tilde{J}_{0}(p d)=\tilde{J}_{0}^{\text {arth }}(p d)-0.15 \cdot\left|\tilde{J}_{0}(p p)-\tilde{J}_{0}(d d)\right|=800.61605 \mathrm{~K} \\
& \tilde{K}_{0}(p d)=\tilde{K}_{0}^{\text {arth }}(p d)+0.02 \cdot\left|\tilde{K}_{0}(p p)-\tilde{K}_{0}(d d)\right|=1484.79504 \mathrm{~K},
\end{aligned}
$$

whereas $(0 ; 0)$ denotes the arithmetic mean set of the parameters.

Figure 4.12 illustrates the composition dependence of transition temperatures in mixed Rochelle salt crystals calculated within a complete theory at different sets of parameters $(u, v)$ given by (4.17). The arithmetic mean set (4.16) yields very weakly non-linear dependences $T_{\text {ci }}(x)$. The more the parameters $\tilde{J}_{0}(p d)$ and $\tilde{K}_{0}(p d)$ deviate from the arithmetic mean set, the more the composition dependence of transition temperatures deviate from the linear one.
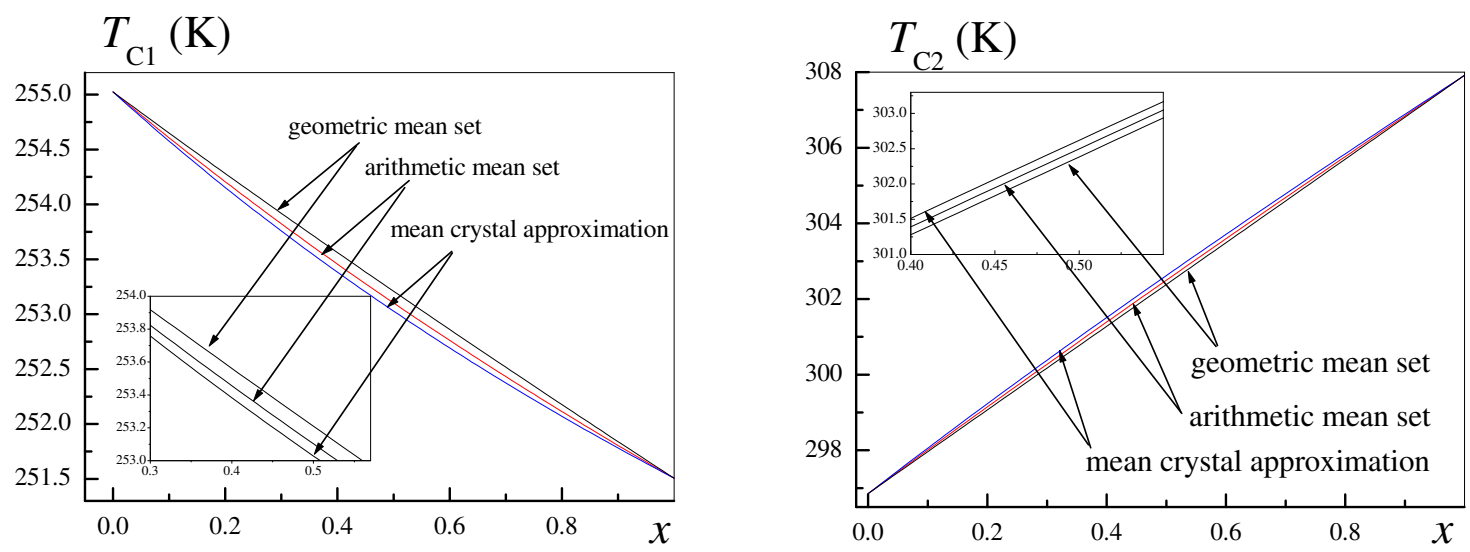

Figure 4.13. Composition dependence $\left(x=x_{d}\right)$ of transition temperatures of mixed Rochelle salt crystals (deuteration levels indicated) calculated within a complete theory with the arithmetic (4.16) and geometric (4.18) mean sets of the theory parameters as well as within the mean crystal approximation (4.15).

In figure 4.13 we show the composition dependence of transition temperatures in mixed Rochelle salt crystals calculated within a complete theory with the arithmetic (4.16) and geometric

$$
\tilde{J}_{0}^{\text {geo }}(p d)=\sqrt{\tilde{J}_{0}(p p) \tilde{J}_{0}(d d)}, \quad \tilde{K}_{0}^{\text {geo }}(p d)=\sqrt{\tilde{K}_{0}(p p) \tilde{K}_{0}(d d)}
$$

mean sets of the theory parameters as well as within the mean crystal approximation (4.15). As one can see, all these methods predict nearly the same slightly non-linear dependences of $T_{\mathrm{ci}}(x)$.

As one can see in figure 4.14, deuteration effects for the other physical characteristics of Rochelle salt predicted by the complete theory qualitatively agree with those given by the mean crystal approximation. 

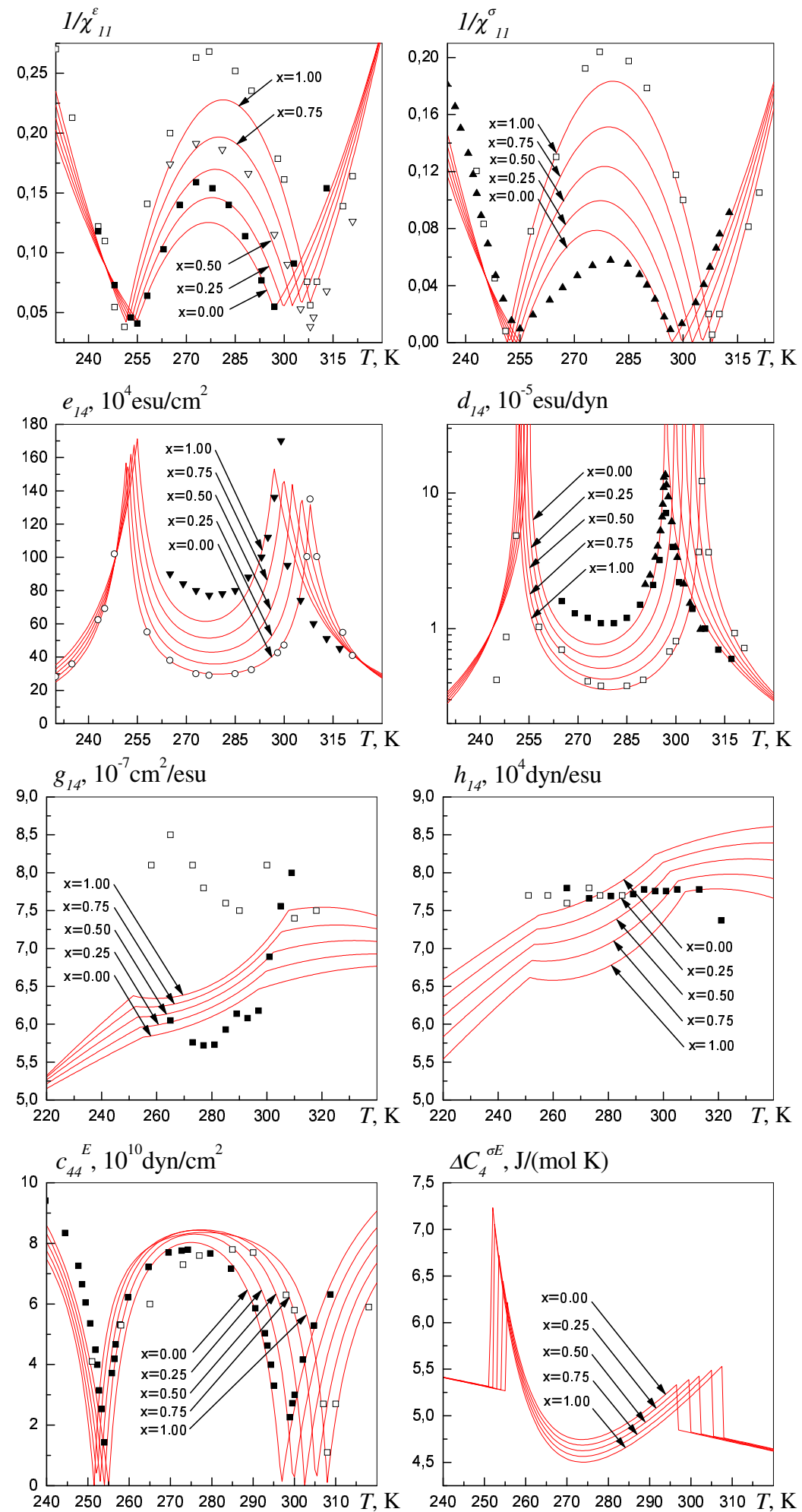

Figure 4.14. Temperature dependences of the physical characteristics of mixed Rochelle salt crystals (deuteration levels $x=x_{d}$ indicated). Lines: a complete theory. Experimental points for $1 / \chi_{11}^{\varepsilon}(T)$ are taken from: $-[12], \square-[46], \nabla-$ [48]; The rest of the points are the same as in figure 4.10. 


\section{Concluding remarks}

Despite the fact that Rochelle salt appears to be the first known ferroelectrics, the mechanism of ferroelectricity in it has not been completely established. In this section we shall analyse the major achievements and miscalculations made in the studies of this type of crystals. It would be useful to compare the history of investigation of ferroelectric crystals with double asymmetric potential with that of the $\mathrm{KH}_{2} \mathrm{PO}_{4}$ family crystals, since both types of crystals are also piezoelectric.

The most prominent peculiarity of the study of the $\mathrm{KH}_{2} \mathrm{PO}_{4}$ family ferroelectrics is a close connection between theory and experiment. Ferroelectricity in $\mathrm{KH}_{2} \mathrm{PO}_{4}$ was discovered in 1938, and already in 1941 the first microscopic theory (Slater model) of the phase transition in it was proposed. Despite certain doubts in its validity, the most important results for these crystals were obtained in the framework of the proton ordering model (see [58]), yielding within a cluster approximation a good description of experimental data for thermodynamic and dynamic characteristics. of the deuterated members of the family. Later, a microscopic theory of deformed deuterated crystals of this type was developed [63], within which a proper description of pressure and electric field effects on their physical characteristics was obtained [59-62]. Essential point in the theoretical studies was a development of the wellgrounded fitting procedure; especially we would like to mention the papers [29-31], where it was shown that in the fitting it is important to have reliable experimental data for the specific heat of the crystals. In our recents works $[64,65]$ we modified the proton ordering model in order to take into account the contributions of piezoelectric coupling, which allowed us to improve the theory and properly describe the experimental data for the elastic, piezoelectric, and dielectric characteristics of the crystals.

There was no such a close connection between theory and experiment in the investigation of the Rochelle salt type ferroelectrics. Due to complexity of the crystal structure of Rochelle salt, there is no much data about the microscopic mechanism of the phase transitions in it. Neither there has been much theoretical effort after the Mitsui model was proposed. The existing theoretical works were either incomplete (only one or very few characteristics were calculated, which does not permit to test the adequacy of the used model) or contradictory in a sense that, for instance, it was impossible to simultaneously obtain a good description of spontaneous polarization and static dielectric permittivity. Qualitatively incorrect were the calculated temperature curves of relaxation times and dynamic dielectric permittivity in the microwave region near the Curie points. Such a situation was to a great extent due to the fact that strong piezoelectric coupling in Rochelle salt was not taken into account and, in fact, the models corresponded to a mechanically free crystal, whereas at microwave frequencies a crystal is effectively clamped (theories of the $\mathrm{KH}_{2} \mathrm{PO}_{4}$ type crystals which do not take into account the piezoelectric effect, yield at least qualitatively correct results due to the first order phase transition in the crystals).

It was fruitful to use an experience obtained in the study of the $\mathrm{KH}_{2} \mathrm{PO}_{4}$ family crystals. Because of a much simpler crystal structure and more detailed experimental 
structural data, the microscopic Hamiltonian with taking into account the piezoelectric coupling for these crystals could be and was derived ab initio [63]. Performing analogous calculations for the Rochelle salt type crystals, we came to a model which allowed us to obtain elastic and piezoelectric characteristics of the crystals, calculate dielectric characteristics of free and clamped crystals, and get a qualitatively (and quantitatively) correct system dynamics near the Curie points $[43,44]$.

An essential insight into the problem of the phase transitions mechanisms in Rochelle salt type crystals can be obtained by investigation of the disordered (partially deuterated) crystals, as it was for the partially deuterated crystals of the $\mathrm{KH}_{2} \mathrm{PO}_{4}$ family, where the essential role in obtaining a proper description of experimental data was played by taking into account the tunneling effects. Availability of extensive experimental data for these crystals for several deuteration levels was strongly helpful. Experimental measurements of the deuteration effects on the physical characteristics of the Rochelle salt type crystals have not been performed yet and are therefore necessary. They would help to elucidate the role played by hydrogen bonds subsystem in the phase transitions in the crystals. In the this work we present the results of theoretical calculations for the partially deuterated Rochelle salt crystals, performed within the mean crystal approximation and within the consistent general theory of the disordered system. Due to the absence of any experimental data for mixed Rochelle salt crystals, we were unable to determine the values of certain fitting parameters and present results obtained with several trial values of those parameters. Verification of the theory predictions is thus strongly required. The obtained theoretical results can possibly be further improved by considering the tunneling effects, electrostriction coupling, as well as by using approximations higher than the mean field appoximation.

\section{Acknowledgement}

This work was supported by the State Foundation for Fundamental Research, project 02.07/00310.

\section{References}

1. Frazer B.C., McKeown M., Pepinsky R. // Phys. Rev., 1954, vol. 94, p. 1435.

2. Jona F., Shirane G. Ferroelectric crystals. Pergamon Press, Oxford-London-New YorkParis, 1962.

3. Iwata Y., Mitani S., Shibuya O. // Ferroelectrics, 1989, vol. 96, p. 215.

4. Iwata Y., Mitani S., Shibuya O. // Ferroelectrics, 1990, vol. 107, p. 287.

5. Volkov A.A., Goncharov Yu.G., Kozlov G.V., Kryukova Ye.B., Petzelt J. // Pis'ma Zh. Eksp. Teor. Fiz., 1985, vol. 41, p. 16. 
6. Volkov A.A., Kozlov G.V., Kryukova Ye.B., Sobyanin A.A. // Fiz. Tverd. Tela, 1986, vol. 28, p. 797 (in Russian).

7. Kamba S., Schaack G., Petzelt J. // Phys. Rev. B, 1995, vol. 51, 14998.

8. Hlinka J., Kulda J., Kamba S., Petzelt J. // Phys. Rev. B, 2001, vol. 63, 052102.

9. Suzuki E., Shiozaki Y.// Phys. Rev. B, 1996, vol. 53, p. 5217.

10. Kulda J., Hlinka J., Kamba S., Petzelt J. The nature of ferroelectric transitions in Rochelle salt. In: ILL: Annual Report 2000. (www.ill.fr/AR-00/p-48.htm)

11. Mitsui T. salt. // Phys. Rev., 1958, vol. 111, No. 5, p. 1529-1567.

12. Sandy F., Jones R.V. // Phys. Rev., 1968, vol. 168, No. 2, p. 481-493.

13. Žekš B., Shukla G.G., Blinc R. // Phys. Rev. B, 1971, vol. 3, p. 2305-2311.

14. Vaks V.G. Introduction into microscopic theory of ferroelectrics. Moscow, Nauka, 1973 (in Russian).

15. Kalenik J. // Acta Phys. Pol., 1975, vol. A48, No 3, p. 387-395.

16. Glauber R.J. // J. Math. Phys., 1963, vol. 4, No. 2, p. 294-307.

17. Žekš B., Shukla G.G., Blinc R. // J.Phys. C, 1972, vol. 33, p. 67-68.

18. Blinc R., Žekš B., Soft modes in ferroelectrics and antiferroelectrics. Elseviers, New York, 1974.

19. Alexandrov K.S., Anistratov A.T. et al.. // Ferroelectrics, 1976, vol. 12, p. 191-193.

20. Blat D.I., Zinenko V.I.. // Fiz. Tverd. Tela, 1976, vol. 18, p. 3599-3604 (in Russian).

21. Vaks V.G., Zinenko V.I., Shneider V.Ye. // Uspekhi Fiz. Nauk, 1983, vol. 141, p. 629673 (in Russian).

22. Levitskii R.R., Verkholyak T.M., Kutny I.V., Hil I.G. Investigation of ferroelectric order-disorder type compounds with asymmetric double-well potential. Preprint condmat/0106351.

23. Dublenych Yu.I. Phase transitions in Mitsui model. Preprint of the Institute for Condensed Matter Physics, ICMP-02-15U, Lviv, 2002, 37 p. (in Ukrainian).

24. Levitskii R.R., Zachek I.R., Varanitskii V.I. // Ukrainian Journal of Physics, 1980, vol. 25, No 11, p. 1766-1774.

25. Levitskii R.R., Zachek I.R., Varanitskii V.I. // Fiz. Tverd. Tela, 1980, vol. 22, p. 27502755 (in Russian).

26. Levitskii R.R., Antonyak Yu.T., Zachek I.R. // Ukrainian Journal of Physics,, 1981, vol. 26, No 11, p. 1835-1838 (in Russian).

27. Levitskii R.R., Grigas J., Paprotny W., Kutny I.V. // Ferroelectrics, 1984, vol. 61, p.1944-1953.

28. Grigas J., Zachek I.R., Krasikov V.S., Kutny I.V. et al., Lithuanian Physics Proceedings, 1984, vol. 24, No 6, p.33-45 (in Russian).

29. Levitskii R.R., Lisnii B.M. // Journ. Phys. Studies, 2002, vol. 6, No 1, p. 91-108.

30. Levitskii R.R., Lisnii B.M., Baran O.R. // Condens. Matter Phys., 2001, vol. 4, No 3, p. $523-552$.

31. Levitskii R.R., Lisnii B.M., Baran O.R. // Condens. Matter Phys., 2002, vol. 5, No 3, p. $553-577$.

32. Stasyuk I.V., Levitskii R.R. // Phys. Stat. Sol. (b), 1970, vol. 39, K35-K38.

33. Stasyuk I.V., Levitskii R.R. // Ukrainian Journal of Physics, 1970, vol. 15, No 3, p. 460-469 (in Russian).

34. Stasyuk I.V., Levitskii R.R. // Bull. Acad. Sci. USSR. Phys. Ser., 1971, vol. 35, No 9, p. 1775-1778 (in Russian).

35. Mori K. // Ferroelectrics, 1981, vol. 31, p.173-178. 
36. Konsin P.I., Kristofel N.N. // Bull. Acad. Sci. USSR. Phys. Ser., 1975, vol. 39, p. 650654 (in Russian).

37. Chaudhuri B.K., Atake T., Ganguli S., Chahara H. // J. Phys. Soc. Jpn, 1980, vol. 49, p. $608-618$.

38. Levitskii R.R., Sorokov S.I. Dynamics of partially deuterated ferroelectric compounds with hydrogen bonds. I. Models of ferroelectrics with asymmetric hydrogen bonds and $\mathrm{PbH}_{1-x} \mathrm{D}_{x} \mathrm{PO}_{4}$. Preprint of the Institute for Theoretical Physics, ITF-78-152R, Kyiv, 1979, 37 p. (in Russian).

39. Belosludov V.R., Vlasova A.A., Nabutovski V.M., Shneider V.E. //, 1979, vol. 77, p. $270-278$.

40. Tornau E.E., Shneider V.E. // Pis'ma Zh. Eksp. Teor. Fiz., 1980, vol. 31, p. 385-388.

41. Vlasova A.A., Stamenkovic S., Tornau E.E., Shneider V.E. // Fiz. Tverd. Tela, 1981, vol. 23, p. 413-417 (in Russian).

42. Levitskii R.R., Sokolovskii R.O. // Condens. Matter Phys., 1999, vol. 2, p.393-400.

43. Levitskii R.R., Zachek I.R., Verkholyak T.M., Moina A.P. // Phys. Rev. B, 2003, vol. 67,1741121

44. Levitskii R.R., Zachek I.R., Verkholyak T.M., Moina A.P. // Condens. Matter Phys., 2003, vol. 6, No 2(34) p. 261-270.

45. Levitskii R.R., Zachek I.R., Moina A.P., Verkholyak T.M. // J. Phys. Studies, 2003, Vol. 7, No 1, p. 106-113.

46. Horioka M., Abe R. // Jap. J. Appl. Phys., 1979, vol. 18, No 11, p. 2065-2071.

47. Hablutzel J. // Helv. Phys. Acta, 1931, vol. 12, p. 489.

48. Holden A.N., Mason W.P. // Phys. Rev. 1940, vol. 57, p. 54.

49. Cady W.G., Piezoelectricity; an introduction to the theory and application of electromechanical phenomena in crystals. New York, London. McGraw Hill Book Company, Inc., 1946.

50. Volkov A.A., Kozlov G.V., Lebedev S.P. // Fiz. Tverd. Tela, 1982, vol. 24, p. 555-561.

51. Kessenikh G.G., Strajblova J., Shuvalov L.A., Janta J. // Czechosl. J. Phys. B, 1973, vol. 23, No 4, p. 495-498.

52. Serdobolskaya O.Yu. // Sol. Stat. Phys., 1996, vol. 38, p. 1529 (in Russian).

53. Gutin L. // Zh. Eksp. Teor. Fiz., 1945, vol. 15, p. 199 (in Russian).

54. Mason W.P. // Phys. Rev., 1939, vol. 55, p. 775.

55. Bronowska W.J. // J. Appl. Crystallogr., 1981, vol. 14, p. 203-207.

56. Taylor W., Lockwood D.J., Labbe H.J. // J. Phys. C.: Solid State Phys., 1984, vol. 17, p. 3685-3699.

57. Beige H., Kühnel A. // Phys. Status Solidi A, 1984, vol. 84, p. 433-437.

58. Tokunaga M., Matsubara T. // Ferroelectrics, 1987, vol. 72, p. 175-191.

59. Stasyuk I.V., Levitskii R.R., Moina A.P. // Phys. Rev. B., 1999, vol. 59, No 13, p. $8530-8540$.

60. Levitskii R.R., Moina A.P., Zachek I.R. // Condens. Matter Phys., 1999, vol. 2, No 3(19), p .515-522.

61. Stasyuk I.V., Levitskii R.R., Moina A.P., Zachek I.R., Duda A.S., Romanyuk M.O., Stadnyk V.J., Shcherbina Ye.V. // Journ. Phys. Studies., 1999, vol. 3, No 4, p. $502-$ 512.

62. Stasyuk I.V., Levitskii R.R., Moina A.P. // Condens. Matter Phys., 1999, vol. 2, No 4(20), p. 731-744.

63. Stasyuk I.V., Biletskii I.N. // Bull. Acad. Sci. USSR. Phys. Ser., 1983, vol. 47, p. 705- 
709 (in Russian).

64. Stasyuk I.V., Levitskii R.R., Zachek I.R., Moina A.P. // Phys. Rev. B., 2000, vol. 62, No 10, p. 6198-6207.

65. Stasyuk I.V., Levitskii R.R., Zachek I.R., Moina A.P., Duda A.S. // Journ. Phys. Studies, 2000, vol. 4. p. 190-201 (in Ukrainian).

\section{Appendix}

$$
\begin{aligned}
& \Delta_{p 1}=\left|\begin{array}{lll}
b_{2} & b_{3} & b_{4} \\
c_{2} & c_{3} & c_{4} \\
d_{2} & d_{3} & d_{4}
\end{array}\right|, \Delta_{p 3}=\left|\begin{array}{ccc}
a_{2} & a_{3} & a_{4} \\
b_{2} & b_{3} & b_{4} \\
d_{2} & d_{3} & d_{4}
\end{array}\right|, \Delta_{p 2}=\left|\begin{array}{ccc}
a_{2} & a_{3} & a_{4} \\
c_{2} & c_{3} & c_{4} \\
d_{2} & d_{3} & d_{4}
\end{array}\right|, \Delta_{p 4}=\left|\begin{array}{lll}
a_{2} & a_{3} & a_{4} \\
b_{2} & b_{3} & b_{4} \\
c_{2} & c_{3} & c_{4}
\end{array}\right|, \\
& \Delta_{d 1}=\left|\begin{array}{lll}
b_{1} & b_{3} & b_{4} \\
c_{1} & c_{3} & c_{4} \\
d_{1} & d_{3} & d_{4}
\end{array}\right|, \Delta_{d 3}=\left|\begin{array}{lll}
a_{1} & a_{3} & a_{4} \\
b_{1} & b_{3} & b_{4} \\
d_{1} & d_{3} & d_{4}
\end{array}\right|, \Delta_{d 2}=\left|\begin{array}{lll}
a_{1} & a_{3} & a_{4} \\
c_{1} & c_{3} & c_{4} \\
d_{1} & d_{3} & d_{4}
\end{array}\right|, \Delta_{d 4}=\left|\begin{array}{lll}
a_{1} & a_{3} & a_{4} \\
b_{1} & b_{3} & b_{4} \\
c_{1} & c_{3} & c_{4}
\end{array}\right|, \\
& \Delta_{4}=\left|\begin{array}{llll}
a_{1} & a_{2} & a_{3} & a_{4} \\
b_{1} & b_{2} & b_{3} & b_{4} \\
c_{1} & c_{2} & c_{3} & c_{4} \\
d_{1} & d_{2} & d_{3} & d_{4}
\end{array}\right|
\end{aligned}
$$

$$
\begin{aligned}
a_{0} & =1-\xi^{2}(p)-\sigma^{2}(p), & c_{0} & =-2 \xi(p) \sigma(p), \\
b_{0} & =1-\xi^{2}(d)-\sigma^{2}(d), & d_{0} & =-2 \xi(d) \sigma(d), \\
a_{1} & =1-\frac{1}{4 T} \tilde{R}^{+}(p p) x_{p}\left[1-\xi^{2}(p)-\sigma^{2}(p)\right], & c_{1} & \left.=\frac{1}{4 T} \tilde{R}^{+}(p p) x_{p} 2 \xi(p) \sigma(p)\right], \\
b_{1} & =-\frac{1}{4 T} \tilde{R}^{+}(p d) x_{p}\left[1-\xi^{2}(d)-\sigma^{2}(d)\right], & d_{1} & \left.=\frac{1}{4 T} \tilde{R}^{+}(p d) x_{p} 2 \xi(d) \sigma(d)\right], \\
a_{2} & =-\frac{1}{4 T} \tilde{R}^{+}(p d) x_{d}\left[1-\xi^{2}(p)-\sigma^{2}(p)\right], & c_{2} & \left.=\frac{1}{4 T} \tilde{R}^{+}(p d) x_{d} 2 \xi(p) \sigma(p)\right], \\
b_{2} & =1-\frac{1}{4 T} \tilde{R}^{+}(d d) x_{d}\left[1-\xi^{2}(d)-\sigma^{2}(d)\right], & d_{2} & \left.=\frac{1}{4 T} \tilde{R}^{+}(d d) x_{d} 2 \xi(d) \sigma(d)\right], \\
a_{3} & \left.=-\frac{1}{4 T} \tilde{R}^{-}(p p) x_{p} 2 \xi(p) \sigma(p)\right], & c_{3} & =1+\frac{1}{4 T} \tilde{R}^{-}(p p) x_{p}\left[1-\xi^{2}(p)-\sigma^{2}(p)\right], \\
b_{3} & \left.=-\frac{1}{4 T} \tilde{R}^{-}(p d) x_{p} 2 \xi(d) \sigma(d)\right], & d_{3} & =\frac{1}{4 T} \tilde{R}^{-}(p d) x_{p}\left[1-\xi^{2}(p)-\sigma^{2}(p)\right], \\
a_{4} & \left.=-\frac{1}{4 T} \tilde{R}^{-}(p d) x_{d} 2 \xi(p) \sigma(p)\right], & c_{4} & =\frac{1}{4 T} \tilde{R}^{-}(p d) x_{d}\left[1-\xi^{2}(p)-\sigma^{2}(p)\right], \\
b_{4} & \left.=-\frac{1}{4 T} \tilde{R}^{-}(d d) x_{d} 2 \xi(d) \sigma(d)\right], & d_{4} & =1+\frac{1}{4 T} \tilde{R}^{-}(d d) x_{d}\left[1-\xi^{2}(d)-\sigma^{2}(d)\right] .
\end{aligned}
$$




\title{
Ізотопічний ефект у частково дейтерованих п'єзоелектричних кристалах сегнетової солі
}

\author{
Р.Р.Левицький ${ }^{1}$, І.Р.Зачек ${ }^{2}$, А.П.Моїна ${ }^{1}$, А.Я.Андрусик ${ }^{1}$ \\ 1 Інститут фізики конденсованих систем, 79011, Львів, вул. \\ Свенціцького, 1 \\ 2 Національний університет “Львівська політехніка”, 79013, Львів, вул. \\ С.Бандери, 12
}

Отримано 4 лютого 2004 р.

Запропоновано теорію діелектричних, п'єзоелектричних і пружних властивостей частково дейтерованих (нерівноважний безлад з повним сортовим хаосом) кристалів сегнетової солі з врахуванням п'єзоелектричних взаємодій. Представлені результати числових розрахунків для повністю дейтерованої сегнетової солі порівнюються з наявними експериментальними даними. Ізотопічний ефект чисельно досліджується в наближенні середнього кристалу та в рамках запропонованої теорії при різних пробних значеннях параметрів моделі. Наведені передбачені теорією для частково дейтерованих систем залежності розрахованих характеристик від температури та рівня дейтерування.

Ключові слова: сегнетова сіль, дейтерування, п'єзоелектричний ефект

PACS: $77.22 . \mathrm{Gm}, 77.65 . \mathrm{Bn}, 77.80 . \mathrm{Bh}$ 
